\title{
Electromagnetic and color memory in even dimensions
}

\author{
Andrea Campoleoni $\odot,{ }^{1, *}$ Dario Francia $\odot,{ }^{2,3, \dagger}$ and Carlo Heissenberg $\oplus^{4, *}$ \\ ${ }^{1}$ Service de Physique de l'Univers, Champs et Gravitation, Université de Mons, \\ Place du Parc, 20 B-7000 Mons, Belgium \\ ${ }^{2}$ Centro Studi e Ricerche E. Fermi, Piazza del Viminale, 1 I-00184 Roma, Italy \\ ${ }^{3}$ Roma Tre University and INFN, Via della Vasca Navale, 84 I-00146 Roma, Italy \\ ${ }^{4}$ Scuola Normale Superiore and INFN, Piazza dei Cavalieri, 7 I-56126 Pisa, Italy
}

(Received 17 July 2019; published 23 October 2019)

\begin{abstract}
We explore memory effects associated with both Abelian and non-Abelian radiation getting to null infinity, in arbitrary even spacetime dimensions. Together with classical memories, linear and nonlinear, amounting to permanent kicks in the velocity of the probes, we also discuss the higher-dimensional counterparts of quantum memory effects, manifesting themselves in modifications of the relative phases describing a configuration of several probes. In addition, we analyze the structure of the asymptotic symmetries of Maxwell's theory in any dimension, both even and odd, in the Lorenz gauge.
\end{abstract}

DOI: 10.1103/PhysRevD.100.085015

\section{INTRODUCTION AND OUTLOOK}

Memory effects are permanent changes in physical quantities pertaining to probes facing the passage of a burst of radiation close to or at null infinity. In this paper we illustrate various types of memories concerning matter probes in interaction with electromagnetic or Yang-Mills radiation, in arbitrary even-dimensional spacetimes.

Following earlier works concerning gravitational memories [1,2], Bieri and Garfinkle [3] first pointed out the existence of analogous observables for electrically charged matter in $D=4$. For charged test particles close to null infinity, they identified the relevant observable in the form of a "velocity kick" that the probes would experience as a consequence of the passage of a radiative perturbation. They also observed, in the same context, that the possibility to source those effects either with matter that does not reach null infinity or with massless sources allows one to identify two types of effects, termed "linear" (or "ordinary") in the former case and "nonlinear" (or "null") in the latter.

Memory effects caused by electromagnetic fields were further discussed in [4] and later connected to asymptotic symmetries of four-dimensional Maxwell's theory, and thus identified as the classical counterparts of soft theorems, in

*andrea.campoleoni@umons.ac.be

Research Associate of the Fund for Scientific Research - FNRS, Belgium.

dario.francia@roma3.infn.it

carlo.heissenberg@sns.it

Published by the American Physical Society under the terms of the Creative Commons Attribution 4.0 International license. Further distribution of this work must maintain attribution to the author(s) and the published article's title, journal citation, and DOI. Funded by SCOAP ${ }^{3}$.
$[5,6]$. See also [7,8]. Moreover, the possible existence of analogous effects in dimension four or higher was investigated from various perspectives, with different (and sometimes not fully compatible) outcomes in [9-15]. Those works adopted various approaches, focused either on the classical solutions to the corresponding wave equations or on the perturbative expansion of the gauge potentials with a suitably assigned boundary behavior.

One of the main goals of this work is to describe ordinary and null memory effects for both Maxwell and Yang-Mills theories in even-dimensional spacetimes. To this end, we inspect the perturbative behavior of the corresponding potentials in the Lorenz gauge and connect it to underlying residual gauge symmetries.

Following $[9,10]$, we begin in Sec. II by analyzing the classical fields generated by specific background currents, so as to construct a number of concrete examples with which to compare our general results of the ensuing sections. In particular, we compute the null memory induced by a charged particle moving at the speed of light on a test particle initially at rest in even dimensions. In Sec. III we discuss various types of memories for Maxwell's theory in even-dimensional spacetimes. Upon employing a recursive gauge-fixing procedure, analogous to the one adopted in [16] in the gravitational setup, we clarify the interpretation of both ordinary and null memory in any even $D$ in terms of residual gauge transformations acting at order $r^{4-D}$. We generalize this procedure to the non-Abelian case in Sec. IV, where we obtain similar results and interpretation for linear and nonlinear memory effects concerning matter in interaction with Yang-Mills radiation.

Both ordinary and null memory effects concern the permanent modifications affecting kinematical properties of the probes (the velocity in the case of spin-one radiation, 
the relative displacement in the gravitational case) due to the interaction of the latter with a transient radiation field close to null infinity. On the other hand, the interaction itself takes place if the matter probes possess the required quantum numbers, electric charge, or color charge, whose overall configuration, whenever several particles are involved, can only be defined by providing the value of the corresponding connection field on the celestial sphere. In this sense, one should expect that the configuration itself be sensitive to the passage of radiation whenever the latter entails a vacuum transition between connections that differ by a (large) gauge transformation, even in the idealized case of unperturbed kinematical variables (i.e., charges kept fixed at specified angles on the sphere). We refer to these types of memory effects as "phase memory" for the electromagnetic case and "color memory" in the nonAbelian setting, to distinguish them from the memory effects affecting kinematical properties of the probes, that we collectively term simply as "electromagnetic" and "Yang-Mills" memories, respectively. To our knowledge, in $D=4$ phase memory was first discussed in [17] while color memory was analyzed in $[18,19]$. Here we explore these phenomena in the higher, even-dimensional case, in Secs. III D and IV D, respectively. Let us observe that, differently from the ordinary and null memories that are purely classical effects, phase memory and color memory naturally arise at the quantum level. It would be interesting to explore possible gravitational counterparts of the color memory effect.

Memory effects in $D>4$ appear at a subleading order with respect to radiation. Correspondingly, their counterparts at the level of gauge transformations do not comprise properly defined infinite-dimensional asymptotic symmetries. The very existence of the latter, on the other hand, was sometimes doubted in the literature because of the need for too-slow falloffs on the gauge potentials that could in principle lead to divergences in physically sensible quantities. The asymptotic structure of spin-one gauge theories, in particular in connection to soft theorems, was widely investigated in the literature [20-33]. More recently, the issue concerning the higher-dimensional extensions of those results was further explored in a number of works [34-37]. On our side, in Sec. V we provide an analysis of the asymptotic symmetries of Maxwell's theory in any $D$, even and odd, stressing analogies and differences between the two cases, always working in the Lorenz gauge. In addition, we describe an explicit procedure for defining the surface charges on $\mathcal{I}$.

In the appendixes we collect a summary of our conventions (Appendix A) together with a number of ancillary results. In Appendix B we provide a detailed discussion of the classical solutions to wave equations and memories for scalar fields in any $D$, comprising among other things the calculation of null memory induced by a charged particle moving at the speed of light on a test particle initially at rest in odd dimensions. In Appendix $\mathrm{C}$ we provide a closedform solution of $\square \epsilon=0$ in any even dimension with boundary condition $\epsilon(u, r, \mathbf{n}) \rightarrow \lambda(\mathbf{n})$ for $r \rightarrow \infty$. In Appendix D we complete our exploration of the infrared triangle for electromagnetism [38] by describing the link between Weinberg's soft theorem and the asymptotic symmetry in the Lorenz gauge, in even dimensions.

\section{CLASSICAL EM SOLUTIONS AND MEMORY EFFECTS}

In this section we calculate the fields generated by specific background charged particles, both massive and massless. In particular, we evaluate in any even $D$ the memory effects induced on a test particle at a large distance $r$ from the origin, much in the spirit of $[9,10,12]$, while also including the effects induced by massless charged particles, thus extending the four-dimensional analysis of [3]. Throughout the paper we shall work in the Lorenz gauge, which in Cartesian coordinates reads $\partial^{\mu} \mathcal{A}_{\mu}=0$, so that the equations of motion reduce to a set of scalar wave equations

$$
\square \mathcal{A}_{\mu}=j_{\mu} .
$$

Thus, much of the calculations are essentially the same as those of the scalar case that we detail in Appendix B.

First, we consider the unphysical but instructive case of a static pointlike source with charge $q$ created at the origin, ${ }^{1}$

$$
\square \mathcal{A}^{\mu}=u^{\mu} q \theta(t) \delta(\mathbf{x}),
$$

where $u^{\mu}=(1,0, \ldots, 0)$. The retarded solution is

$$
\mathcal{A}^{\mu}=-u^{\mu} \varphi,
$$

where $\varphi$ denotes the corresponding solution (B7) for the scalar field that we report here for completeness,

$$
\varphi(u, r)=q \sum_{k=0}^{D / 2-2} c_{D, k} \frac{\theta^{(D / 2-2-k)}(u)}{r^{D / 2-1+k}} .
$$

Here $\theta(u)$ denotes the Heaviside distribution, while the coefficients $c_{D, k}$ are specified in (B5). The coordinates $u$ and $r$ are part of the retarded Bondi coordinates, in which the Minkowski metric takes the form

$$
d s^{2}=-d u^{2}-2 d u d r+r^{2} \gamma_{i j} d x^{i} d x^{j} .
$$

The solution describing a source created at the origin with velocity $\mathbf{v}$ is obtained by boosting the previous one

\footnotetext{
${ }^{1}$ Strictly speaking, Eq. (2.2) is not well posed, since the righthand side has a nonzero divergence. We shall take this aspect in due account when calculating the solution (2.7), where in particular the right-hand side of (2.2) occurs just as part of a full source that respects the continuity equation.
} 
according to (B12) and yields $\mathcal{A}^{\mu}=\left(\mathcal{A}^{0}, \mathbf{A}\right)=-\gamma(\mathbf{v})(1, \mathbf{v}) \varphi$. Moving to retarded coordinates gives

$$
\mathcal{A}_{u}=\gamma(\mathbf{v}) \varphi, \quad \mathcal{A}_{r}=\gamma(\mathbf{v})(1-\mathbf{n} \cdot \mathbf{v}) \varphi, \quad \mathcal{A}_{i}=-r \gamma(\mathbf{v}) v_{i} \varphi,
$$

where $\mathbf{n}=\mathbf{n}\left(x^{i}\right)$ is the unit vector identifying points on the $(D-2)$-sphere and $v_{i}=\mathbf{v} \cdot \partial_{i} \mathbf{n}$.

Focusing for simplicity on the case of $D=6$, let us now consider the radiation field generated by a massive particle with charge $q$ sitting at rest in the origin for $t<0$ that starts moving with velocity $\mathbf{v}$ at $t=0$. Such a field is obtained by matching the solution for a charge destroyed at the origin to that of a charge there created with velocity v. Proceeding as described in Appendix B, one obtains, for large values of $r$,

$$
\begin{aligned}
8 \pi^{2} \mathcal{A}_{u} & =\frac{q \delta(u)}{r^{2}}\left(\frac{1}{1-\mathbf{n} \cdot \mathbf{v}}-1\right)+\mathcal{O}\left(r^{-3}\right), \\
8 \pi^{2} \mathcal{A}_{r} & =\mathcal{O}\left(r^{-3}\right), \\
8 \pi^{2} \mathcal{A}_{i} & =-\frac{q v_{i} \delta(u)}{(1-\mathbf{n} \cdot \mathbf{v}) r}-\frac{q v_{i} \theta(u)}{\gamma(\mathbf{v})^{2}(1-\mathbf{n} \cdot \mathbf{v})^{3} r^{2}}+\mathcal{O}\left(r^{-3}\right),
\end{aligned}
$$

where we kept the orders relevant to the calculation of the memory effect. The change in the angular components of the momentum $P_{\mu}$ of a test charge $Q$, initially at rest, gives rise to a linear (or ordinary) memory effect that, to leading order, reads

$$
\begin{aligned}
\left.P_{i}\right|_{u>0}-\left.P_{i}\right|_{u<0} & =Q \int_{-\infty}^{+\infty} \mathcal{F}_{i u} d u^{\prime} \\
& =\frac{q Q v_{i}\left(2-\mathbf{v}^{2}-\mathbf{n} \cdot \mathbf{v}\right)}{8 \pi^{2}(1-\mathbf{n} \cdot \mathbf{v})^{3} r^{2}}+\mathcal{O}\left(r^{-3}\right),
\end{aligned}
$$

where $\mathcal{F}_{\mu \nu}$ is the Maxwell field strength.

With hindsight, having in mind in particular the results of [10], in order to interpret this leading memory effect in terms of a symmetry, it is useful to perform a further gauge fixing in order to get rid of the residual symmetries affecting the radiation order. Choosing a gauge parameter

$$
8 \pi^{2} \epsilon=-\frac{q \theta(u)}{r^{2}}\left(\frac{1}{1-\mathbf{n} \cdot \mathbf{v}}-1\right)+\mathcal{O}\left(r^{-3}\right)
$$

allows us to cancel the leading term of $\mathcal{A}_{u}$ compatibly with the condition $\square \epsilon=0$. The resulting field after performing this gauge transformation satisfies ${ }^{2}$

\footnotetext{
${ }^{2}$ More explicitly, one has $\mathcal{A}_{u}=\frac{q}{8 \pi^{2} r^{3}}+\mathcal{O}\left(r^{-4}\right)$.
}

$$
\begin{aligned}
\mathcal{A}_{u} & =\mathcal{O}\left(r^{-3}\right), \\
8 \pi^{2} \mathcal{A}_{i} & =-\frac{q v_{i} \delta(u)}{(1-\mathbf{n} \cdot \mathbf{v}) r}-\frac{q v_{i} \theta(u)\left(2-\mathbf{v}^{2}-\mathbf{n} \cdot \mathbf{v}\right)}{(1-\mathbf{n} \cdot \mathbf{v})^{3} r^{2}}+\mathcal{O}\left(r^{-3}\right),
\end{aligned}
$$

so that, in particular, $\mathcal{A}_{i}$ is fully responsible for the memory formula (2.8). The effect is proportional to the variation of the latter field component between $u>0$ and $u<0$ and takes the form of a total derivative on the sphere, close to null infinity:

$$
\begin{aligned}
\left.\mathcal{A}_{i}\right|_{u>0}-\left.\mathcal{A}_{i}\right|_{u<0}= & -\frac{q v_{i}\left(2-\mathbf{v}^{2}-\mathbf{n} \cdot \mathbf{v}\right)}{8 \pi^{2}(1-\mathbf{n} \cdot \mathbf{v})^{3} r^{2}}+\mathcal{O}\left(r^{-3}\right) \\
= & -\frac{q}{8 \pi^{2} r^{2}} \partial_{i}\left(\frac{1-\mathbf{v}^{2}}{2(1-\mathbf{n} \cdot \mathbf{v})^{2}}+\frac{1}{1-\mathbf{n} \cdot \mathbf{v}}\right) \\
& +\mathcal{O}\left(r^{-3}\right) .
\end{aligned}
$$

This result provides an explicit connection between the memory effect and a residual symmetry acting, for large values of $r$, at Coulombic order. ${ }^{3}$

Let us turn our attention to the case of null memory. The four-dimensional case was illustrated in [3]. Let us consider, in any even dimension $D \geq 6$, the field generated by a charge moving in the $\hat{\mathbf{x}}$ direction at the speed of light: in Cartesian coordinates,

$$
\square \mathcal{A}^{\mu}=q v^{\mu} \delta(\mathbf{x}-\hat{\mathbf{x}} t),
$$

with $v^{\mu}=(1, \hat{\mathbf{x}})$ and $|\hat{\mathbf{x}}|=1$. Taking into account the corresponding retarded solution (B24) for the scalar field $\varphi$, we then have $A^{\mu}=-v^{\mu} \varphi$ and, moving to retarded coordinates,

$$
\mathcal{A}_{u}=\varphi, \quad \mathcal{A}_{r}=(1-\mathbf{n} \cdot \hat{\mathbf{x}}) \varphi, \quad \mathcal{A}_{i}=-r \hat{x}_{i} \varphi .
$$

Consequently

$\mathcal{A}_{u} \sim-\frac{q \delta(u)}{r^{D-4}}(\Delta-D+4)^{-1}(\mathbf{n}, \hat{\mathbf{x}})$,

$\mathcal{A}_{r} \sim-\frac{q \delta(u)}{r^{D-4}}\left(1-\mathbf{n} \cdot \mathbf{x}_{0}\right)(\Delta-D+4)^{-1}(\mathbf{n}, \hat{\mathbf{x}})$,

$\mathcal{A}_{i} \sim \frac{q \hat{x}_{i} \delta(u)}{r^{D-5}}(\Delta-D+4)^{-1}(\mathbf{n}, \hat{\mathbf{x}})$,

where $(\Delta-D+4)^{-1}$ denotes the Green function for the operator $\Delta-D+4$ and we have omitted terms of the form

$$
\frac{\delta^{(D-4-k)}(u)}{r^{k}} F(\mathbf{n})
$$

\footnotetext{
${ }^{3}$ The Coulombic order is identified by the condition that the modulus of the vector $\mathcal{A}_{\mu}$ in the given metric scales as $\mathcal{O}\left(r^{3-D}\right)$. In particular, at this order, $\mathcal{A}_{i} \sim \mathcal{O}\left(r^{4-D}\right)$.
} 
[see (B24)], which would be leading with respect to those displayed in (2.14), for suitable values of $k$, but which do not contribute to the memory effect because they integrate to zero. The null memory formula then reads

$$
\begin{aligned}
\left.P_{i}\right|_{u>0}-\left.P_{i}\right|_{u<0} & =Q \int_{-\infty}^{+\infty} \mathcal{F}_{i u} d u^{\prime} \\
& =-\frac{q Q}{r^{D-4}} \partial_{i}(\Delta-D+4)^{-1}(\mathbf{n}, \hat{\mathbf{x}}),
\end{aligned}
$$

where this result is exact for the solution considered, while for more general solutions of the wave equation in the form (B23) it would provide the leading-order contribution. For any even dimension $D>4$, the Green functions above can be written explicitly as follows: denoting for brevity $k=\frac{D-4}{2}$,

$$
\begin{aligned}
& (\Delta-2 k)^{-1}(\mathbf{n}, \hat{\mathbf{x}}) \\
& =-\frac{\Gamma(k+1)}{8 k \pi^{k+1}\left(1-(\mathbf{n} \cdot \hat{\mathbf{x}})^{2}\right)^{k}}\left[1+\frac{\int_{0}^{\mathbf{n} \cdot \hat{\mathbf{x}}}\left(1-t^{2}\right)^{k-1} d t}{\int_{0}^{1}\left(1-t^{2}\right)^{k-1} d t}\right] .
\end{aligned}
$$

Using this formula, it is interesting to remark that, for $D=6$ (i.e., $k=1$ ), the massless limit $\mathbf{v} \rightarrow \hat{\mathbf{x}}$ of the ordinary memory effect (2.8) coincides with the null memory effect (2.16) and correspondingly the gauge field undergoes the leading-order shift

$$
\left.\mathcal{A}_{i}\right|_{u>0}-\left.\mathcal{A}_{i}\right|_{u<0}=-\frac{q}{8 \pi^{2} r^{2}} \partial_{i}\left(\frac{1}{1-\mathbf{n} \cdot \hat{\mathbf{x}}}\right) .
$$

\section{ELECTROMAGNETIC MEMORY}

In the previous section we saw how to establish a connection between local symmetries acting at large $r$ and memory effects for specific matter configurations. Now we would like to investigate this connection beyond those examples by studying the general structure of the solution space in the Lorenz gauge. To this end, we will analyze the asymptotic behavior of the fields in a powerseries expansion in the radial coordinate in $D>6$, which is sufficient to the description of the memory effects. More general possibilities, also encompassing the four-dimensional case, will be discussed in Sec. V, where we will explore the full structure of the asymptotic symmetries in the Lorenz gauge.

\section{A. Electromagnetism in the Lorenz gauge}

The Lorenz gauge condition reads

$\nabla^{\mu} \mathcal{A}_{\mu}=-\partial_{u} \mathcal{A}_{r}+\frac{1}{r}\left(r \partial_{r}+D-2\right)\left(\mathcal{A}_{r}-\mathcal{A}_{u}\right)+\frac{1}{r^{2}} \mathcal{D}^{i} \mathcal{A}_{i}=0$, where $\mathcal{D}_{i}$ is the covariant derivative on the Euclidean unit $(D-2)$-sphere with metric $\gamma_{i j}$. The residual symmetry parameters then satisfy $\square \epsilon=0$, namely

$$
\left(2 \partial_{r}+\frac{D-2}{r}\right) \partial_{u} \epsilon=\left(\partial_{r}^{2}+\frac{D-2}{r} \partial_{r}+\frac{1}{r^{2}} \Delta\right) \epsilon .
$$

The equations of motion reduce to $\square \mathcal{A}_{\mu}=0$, which, component by component, read

$$
\begin{gathered}
{\left[\partial_{r}^{2}-2 \partial_{u} \partial_{r}-\frac{D-2}{r}\left(\partial_{u}-\partial_{r}\right)+\frac{1}{r^{2}} \Delta\right] \mathcal{A}_{u}=0} \\
{\left[\partial_{r}^{2}-2 \partial_{u} \partial_{r}-\frac{D-2}{r}\left(\partial_{u}-\partial_{r}\right)+\frac{1}{r^{2}} \Delta\right] \mathcal{A}_{r}} \\
\quad+\frac{D-2}{r^{2}}\left(\mathcal{A}_{u}-\mathcal{A}_{r}\right)-\frac{2}{r^{3}} \mathcal{D} \cdot \mathcal{A}=0 \\
{\left[\left(\partial_{r}^{2}-2 \partial_{u} \partial_{r}+\frac{1}{r^{2}} \Delta\right)-\frac{D-4}{r}\left(\partial_{u}-\partial_{r}\right)-\frac{D-3}{r^{2}}\right] \mathcal{A}_{i}} \\
\quad-\frac{2}{r} \mathcal{D}_{i}\left(\mathcal{A}_{u}-\mathcal{A}_{r}\right)=0
\end{gathered}
$$

\section{B. Asymptotic expansion}

We assume an asymptotic expansion of the gauge field and of the gauge parameters in powers of $1 / r$

$$
\mathcal{A}_{\mu}=\sum_{k} A_{\mu}^{(k)} r^{-k}, \quad \epsilon=\sum_{k} \epsilon^{(k)} r^{-k},
$$

where the summation ranges are, for the moment, unspecified. Equations (3.1) and (3.2) then give

$\partial_{u} A_{r}^{(k+1)}=(k-D+2)\left(A_{u}^{(k)}-A_{r}^{(k)}\right)+\mathcal{D} \cdot A^{(k-1)}$,
$(D-2 k-2) \partial_{u} \epsilon^{(k)}=[\Delta+(k-1)(k-D+2)] \epsilon^{(k-1)}$,

while from (3.3) one obtains

$$
\begin{aligned}
(D-2 k-2) \partial_{u} A_{u}^{(k)}= & {[\Delta+(k-1)(k-D+2)] A_{u}^{(k-1)}, } \\
(D-2 k-2) \partial_{u} A_{r}^{(k)}= & {[\Delta+k(k-D+1)] A_{r}^{(k-1)} } \\
+ & (D-2) A_{u}^{(k-1)}-2 \mathcal{D} \cdot A^{(k-2)}, \\
(D-2 k-4) \partial_{u} A_{i}^{(k)}= & {[\Delta+k(k-D+3)-1] A_{i}^{(k-1)} } \\
& -2 \mathcal{D}_{i}\left(A_{u}^{(k)}-A_{r}^{(k)}\right) .
\end{aligned}
$$

Equations (3.5)-(3.9) appear in particular to order $r^{-(k+1)}$ in the asymptotic expansions of the original equations.

As we shall see, for the purpose of analyzing the electromagnetic memory effects the leading falloffs can be chosen so as to match the corresponding radiation falloffs: 


$$
\begin{aligned}
\mathcal{A}_{u} & =\mathcal{O}\left(r^{-(D-2) / 2}\right), \quad \mathcal{A}_{r}=\mathcal{O}\left(r^{-(D-2) / 2}\right), \\
\mathcal{A}_{i} & =\mathcal{O}\left(r^{-(D-4) / 2}\right) .
\end{aligned}
$$

More general options are possible and influence the structure of the asymptotic symmetry group. We will be concerned with these more general aspects of the discussion in Sec. V.

The significance of the choice (3.10) lies in the fact that the derivatives with respect to $u$ of the field components are unconstrained to leading order: these conditions for the asymptotic expansion are well suited for identifying the boundary data for a radiation solution with an arbitrary wave form. Such components also provide the energy flux at a given retarded time, according to

$$
\mathcal{P}(u)=\int_{S_{u}} \gamma^{i j} \partial_{u} A_{i}^{\left(\frac{D-4}{2}\right)} \partial_{u} A_{j}^{\left(\frac{D-4}{2}\right)} d \Omega,
$$

where $S_{u}$ is the section of $\mathcal{I}^{+}$at fixed $u$ and $d \Omega$ is the measure element on the unit $(D-2)$-sphere.

The asymptotic behavior of radiation differs in higher dimensions with respect to the characteristic Coulombic falloff $r^{3-D}$ that, in its turn, can be identified as the leading falloff for stationary solutions. (See also the discussion in Sec. III C on this point.) As we shall see, Coulomb fields give nonvanishing contributions to the surface integral associated with the electric charge as well as to the memory effects.

\section{Recursive gauge fixing}

The gauge variations

$$
\begin{aligned}
& \delta A_{u}^{(k)}=\partial_{u} \epsilon^{(k)}, \quad \delta A_{r}^{(k)}=-(k-1) \epsilon^{(k-1)}, \\
& \delta A_{i}^{(k)}=\partial_{i} \epsilon^{(k)}
\end{aligned}
$$

imply a number of restrictions on the allowed gauge parameters, in order to keep the corresponding falloffs. From $\delta \mathcal{A}_{r}=\mathcal{O}\left(r^{-\frac{D-2}{2}}\right)$ we read off $\epsilon^{(k)}=0$ for $k<(D-4) / 2$ and $k \neq 0$, while $\delta A_{u}^{(k)}=\mathcal{O}\left(r^{-\frac{D-2}{2}}\right)$ additionally requires that $\epsilon^{\left(\frac{D-4}{2}\right)}$ be independent of $u$, whereas $\delta A_{i}^{(k)}$ does not give rise to further constraints at this stage. This leads to a gauge parameter of the following form:

$$
\epsilon\left(u, r, x^{i}\right)=1+r^{-\frac{D-4}{2}} \epsilon^{\left(\frac{D-4}{2}\right)}\left(x^{i}\right)+\mathcal{O}\left(r^{-\frac{D-2}{2}}\right),
$$

where we conventionally set the global part of $\epsilon$ to 1 . However, by (3.6),

$$
\left[\Delta-\frac{(D-2)(D-4)}{4}\right] \epsilon^{\left(\frac{D-4}{2}\right)}=0,
$$

which implies $\epsilon^{\left(\frac{D-4}{2}\right)}=0$ since the Laplacian on the sphere is negative semidefinite. ${ }^{4}$ Thus, we need to search further down in the asymptotic expansion of $\epsilon$, employing a recursive on-shell gauge-fixing procedure. The residual symmetry is parametrized as follows:

$$
\epsilon\left(u, r, x^{i}\right)=1+r^{-\frac{D-2}{2}} \epsilon^{\left(\frac{D-2}{2}\right)}\left(u, x^{i}\right)+\cdots .
$$

Equation (3.6) leaves the $u$ dependence of $\epsilon^{\left(\frac{D-2}{2}\right)}$ unconstrained, and therefore we may use it to set

$$
A_{u}^{\left(\frac{D-2}{2}\right)}=0
$$

leaving a residual parameter $\epsilon^{\left(\frac{D-2}{2}\right)}\left(x^{i}\right)$ arbitrary. Setting $k=D / 2$, Eqs. (3.7) and (3.6) reduce to

$\partial_{u} A_{u}^{\left(\frac{D}{2}\right)}=0, \quad \partial_{u} \epsilon^{\left(\frac{D}{2}\right)}+\left[\Delta-\frac{(D-2)(D-4)}{4}\right] \epsilon^{\left(\frac{D-2}{2}\right)}=0$,

respectively. Thus, $A_{u}^{\left(\frac{D}{2}\right)}$ is a function of the angles $x^{i}$ only, while $\delta A_{u}^{\left(\frac{D}{2}\right)}=\partial_{u} \epsilon^{\left(\frac{D}{2}\right)}$ can be expressed in terms of $\epsilon^{\left(\frac{D-2}{2}\right)}$, which can be used to set $A_{u}^{\left(\frac{D}{2}\right)}=0$, while still leaving $\epsilon^{\left(\frac{D}{2}\right)}\left(x^{i}\right)$ arbitrary. We proceed recursively, assuming

$A_{u}^{\left(\frac{D-2}{2}\right)}=A_{u}^{\left(\frac{D}{2}\right)}=\cdots=A_{u}^{(q-1)}=0, \quad \epsilon^{(q-1)}\left(x^{i}\right)$ arbitrary,

for some $q>D / 2$. Then, for $k=q-1$, Eqs. (3.7) and (3.6) give

$$
\begin{aligned}
(D-2 q-2) \partial_{u} A_{u}^{(q)} & =0, \\
(D-2 q-2) \partial_{u} \epsilon^{(q)} & =[\Delta-(q-1)(D-q-2)] \epsilon^{(q-1)} .
\end{aligned}
$$

Therefore, we may employ $\epsilon^{(q-1)}\left(x^{i}\right)$ to set $A_{u}^{(q)}$ to zero provided that the differential operator on the right-hand side is invertible, which is true for any $q<D-2$.

We shall now consider two options. We may first choose to truncate the recursive gauge fixing right after the step labeled by $q=D-4$, which leaves us with the asymptotic expansions

\footnotetext{
${ }^{4}$ Interestingly, however, let us observe that the corresponding putative charge

$$
\tilde{\mathcal{Q}}_{\epsilon}=\lim _{r \rightarrow \infty} \int_{S_{u}} \epsilon \mathcal{F}_{u r} r^{D-2} d \Omega=\int \mathcal{D} \cdot A^{\left(\frac{D-4}{2}\right)} \epsilon^{\left(\frac{D-4}{2}\right)} d \Omega
$$

is finite and nonvanishing as $r \rightarrow \infty$.
} 
$\mathcal{A}_{u}=\sum_{k=D-3}^{\infty} A_{u}^{(k)} r^{-k}, \quad \mathcal{A}_{r}=\sum_{k=\frac{D}{2}}^{\infty} A_{r}^{(k)} r^{-k}, \quad \mathcal{A}_{i}=\sum_{k=\frac{D-4}{2}}^{\infty} A_{i}^{(k)} r^{-k}$,

where $A_{r}^{\left(\frac{D-2}{2}\right)}=0$ on shell. The residual symmetry is then given by

$$
\epsilon\left(u, r, x^{i}\right)=1+\epsilon^{(D-4)}\left(x^{i}\right) r^{4-D}+\cdots,
$$

whose corresponding charge, evaluated in the absence of radiation close to the past boundary $\mathcal{I}_{-}^{+}$of $\mathcal{I}^{+}$, reads ${ }^{5}$

$\mathcal{Q}_{\epsilon}=\frac{1}{r^{D-4}} \int_{\mathcal{I}_{ \pm}^{ \pm}}\left(\partial_{u} A_{r}^{(D-2)}+(D-3) A_{u}^{(D-3)}\right) \epsilon^{(D-4)} d \Omega_{D-2}$.

Alternatively, we may also perform the recursive gauge fixing until the very last allowed step, $q=D-3$, thus obtaining

$$
\begin{aligned}
\mathcal{A}_{u} & =\sum_{k=D-2}^{\infty} A_{u}^{(k)} r^{-k}, \quad \mathcal{A}_{r}=\sum_{k=\frac{D}{2}}^{\infty} A_{r}^{(k)} r^{-k}, \\
\mathcal{A}_{i} & =\sum_{k=\frac{D-4}{2}}^{\infty} A_{i}^{(k)} r^{-k}
\end{aligned}
$$

and

$$
\epsilon\left(u, r, x^{i}\right)=1+\epsilon^{(D-3)}\left(x^{i}\right) r^{3-D}+\cdots .
$$

The latter choice highlights the possibility of making the components

$$
A_{i}^{\left(\frac{D-4}{2}\right)}, \ldots, A_{i}^{(D-5)}, A_{i}^{(D-4)}
$$

gauge invariant, and hence in principle responsible for any observable effect due to radiation impinging on a test charge placed at a large distance $r$ from a source. Indeed, consistently with the examples of Sec. II, in the next section we shall see that electromagnetic memory effects appear at the Coulombic order $A_{i}^{(D-4)}$.

\section{Electromagnetic memory}

A test particle with charge $Q$, initially at rest at a large distance $r$ from the origin, will experience a leading-order momentum kick due to the presence of an electric field according to

\footnotetext{
${ }^{5}$ Similar considerations will apply to the evaluation of soft charges in Sec. V.
}

$$
\left.P_{i}\right|_{u_{1}}-\left.P_{i}\right|_{u_{0}}=Q \int_{u_{0}}^{u_{1}} \mathcal{F}_{i u} d u
$$

We are neglecting the contribution from the magnetic field, assuming the interaction time between the test particle and the radiation is sufficiently small. In particular, Eq. (3.26) holds to leading order for an ideally sharp wave front, as we checked in Sec. II.

Now we shall consider solutions that are stationary before $u=u_{0}$ and after $u=u_{1}$. For such solutions, the Maxwell tensor does not vanish outside this interval, in general, because static forces are present. However, it does not contain radiation, and thus all the components of the gauge potential associated with radiation are to vanish, or, more generally, are to be pure gauge. In particular, the radiation field components before $u_{0}$ and after $u_{1}$ are to be identical or are to differ by a gauge transformation.

Let us combine the information of the previous recursive gauge fixing with the requirement that the solution be stationary before $u_{0}$ and after $u_{1}$. The coefficients $F_{i u}^{(k)}$ of the asymptotic expansion of the electric field $\mathcal{F}_{i u}=$ $\partial_{u} \mathcal{A}_{i}-\partial_{i} \mathcal{A}_{u}$ satisfy

$$
F_{i u}^{(k)}=-\partial_{u} A_{i}^{(k)}\left(u, x^{k}\right),
$$

for $k=\frac{D-4}{2}, \ldots, D-4$ since the corresponding $A_{u}^{(k)}$ are zero, thanks to the gauge fixing. Thus,

$\left.P_{i}\right|_{u_{1}}-\left.P_{i}\right|_{u_{0}}=-Q \sum_{k=\frac{D-4}{2}}^{D-4} \frac{1}{r^{k}}\left(\left.A_{i}^{(k)}\right|_{u_{1}}-\left.A_{i}^{(k)}\right|_{u_{0}}\right)+\mathcal{O}\left(r^{3-D}\right)$.

With respect to our previous observation, let us notice that the components of $\mathcal{A}_{i}$ that enter the subleading terms $\mathcal{O}\left(r^{3-D}\right)$ are those connected with nonvanishing stationary properties of the field (we assume no long-range magnetic forces), while all the leading components explicitly written enter the radiation behavior and thus their difference after $u_{1}$ and before $u_{0}$ can be at most the angular gradient of given functions. In addition, we shall immediately see that, combining this information with the equations of motion will allow us to appreciate that they all vanish with the exception of the last one at order $r^{4-D}$.

Indeed, let us note that for a stationary solution, in our gauge, Eqs. (3.5), (3.7), (3.8), and (3.9) read, for $k<D-2$,

$$
(D-k-1)\left(A_{r}^{(k-1)}-A_{u}^{(k-1)}\right)+\mathcal{D} \cdot A^{(k-2)}=0,
$$

and

$$
[\Delta+(k-1)(k-D+2)] A_{u}^{(k-1)}=0
$$




$$
[\Delta+k(k-D+1)] A_{r}^{(k-1)}+(D-2) A_{u}^{(k-1)}-2 \mathcal{D} \cdot A^{(k-2)}=0,
$$

$$
[\Delta+(k-1)(k-D+2)-1] A_{i}^{(k-2)}-2 \mathcal{D}_{i}\left(A_{u}^{(k-1)}-A_{r}^{(k-1)}\right)=0 .
$$

For $1<k<D-2$, Eq. (3.30) implies $A_{u}^{(k-1)}=0$ consistently with the recursive gauge fixing. Equations (3.29) and (3.31) then give, for $1<k<D-2$,

$$
[\Delta+(k-2)(k-D+1)] A_{r}^{(k-1)}=0
$$

so that $A_{r}^{(k-1)}=0$ for $2<k<D-2$. Considering finally Eq. (3.32), for $2<k<D-2$, we have

$$
[\Delta+(k-1)(k-D+2)-1] A_{i}^{(k-2)}=0,
$$

and hence $A_{i}^{(k-2)}=0$ provided that $k$ also satisfies $k_{-}(D)<k<k_{+}(D)$ with

$$
k_{ \pm}(D)=\frac{1}{2}\left[D-1 \pm \sqrt{(D-3)^{2}+4}\right]
$$

actually, $k_{-}(D)<1$ and $k_{+}(D)>D-2$ for any $D>3$, so we conclude that stationary solutions obey $A_{i}^{(k-2)}=0$ for $2<k<D-2$.

To summarize, $A_{u}^{(k)}=0$ for $0<k<D-3$, while $A_{r}^{(k)}=0$ for $1<k<D-3$ and

$$
A_{i}^{(k)}=0 \text { for } 0<k<D-4 .
$$

By Eq. (3.28), the condition on $\mathcal{A}_{i}$ implies that the leadingorder memory effect appears at $\mathcal{O}\left(r^{4-D}\right)$,

$\left.P_{i}\right|_{u_{1}}-\left.P_{i}\right|_{u_{0}}=-\frac{Q}{r^{D-4}}\left(\left.A_{i}^{(D-4)}\right|_{u_{1}}-\left.A_{i}^{(D-4)}\right|_{u_{0}}\right)+\mathcal{O}\left(r^{3-D}\right)$.

In view of the discussion below (3.28), we conclude that the momentum shift must take the form

$$
\left.P_{i}\right|_{u_{1}}-\left.P_{i}\right|_{u_{0}}=\frac{Q}{r^{D-4}} \partial_{i} g\left(x^{k}\right)+\mathcal{O}\left(r^{3-D}\right),
$$

where $g\left(x^{i}\right)$ is a $u$-independent function, which depends on the shape of the radiation train and in particular on $u_{0}$ and $u_{1}$ [see the example (2.11)].

Let us note that, as it must be, this difference is not affected by the action of the residual gauge transformation (3.21), because the latter is $u$ independent and thus cannot alter the difference $\left.A_{i}^{(D-4)}\right|_{u_{1}}-\left.A_{i}^{(D-4)}\right|_{u_{0}}$. In this sense, whether one performs the last step of the recursive gauge fixing is irrelevant to the extent of calculating the electromagnetic memory.
To conclude, we have established a formula that, for any even $D$, exhibits a momentum kick characterizing the transition between the initial and final vacuum configurations, parametrized by the gauge transformation $g\left(x^{i}\right)$, induced by the exposure to electromagnetic radiation reaching null infinity. In particular, the norm of this effect scales as $r^{3-D}$.

Up to this point we have only been dealing with an ordinary memory effect; in order to encompass null memory, we must modify the equations of motion (3.3) by adding a suitable source term to the right-hand side, namely a current density $J^{\mu}$ allowing for the outflow to future null infinity of charged massless particles. The falloff conditions on such a current can be taken as follows:

$$
J_{u}=\mathcal{O}\left(r^{2-D}\right), \quad J_{r}=\mathcal{O}\left(r^{2-D}\right), \quad J_{i}=\mathcal{O}\left(r^{3-D}\right) .
$$

This is clearly displayed by the current for a single massless charge $q$ moving in the $\hat{\mathbf{x}}$ direction, whose components read in Minkowski coordinates

$$
J^{0}=q \delta(\mathbf{x}-\hat{\mathbf{x}} t), \quad \mathbf{J}=q \hat{\mathbf{x}} \delta(\mathbf{x}-\hat{\mathbf{x}} t),
$$

and in retarded coordinates (for $t=u+r>0$ )

$$
\begin{aligned}
J_{u} & =-\frac{q}{r^{D-2}} \delta(u) \delta(\mathbf{n}, \hat{\mathbf{x}}), \\
J_{r} & =-\frac{q(1-\mathbf{n} \cdot \hat{\mathbf{x}})}{r^{D-2}} \delta(u) \delta(\mathbf{n}, \hat{\mathbf{x}}), \\
J_{i} & =\frac{q \hat{x}_{i}}{r^{D-3}} \delta(u) \delta(\mathbf{n}, \hat{\mathbf{x}}) .
\end{aligned}
$$

To the purposes of the recursive gauge fixing, the only modification is thus the introduction of a source term $J_{u}^{(D-2)}$ in the right-hand side of (3.7) when $k=D-3$, which now actually forces us to stop the gauge fixing after the use of the said equation for $k=D-4$ (the step labeled by $q=$ $D-4$ in the previous section) and leaves us with the falloff (3.20). On the contrary, reaching (3.23) is not allowed, and thus (3.20) comprises a complete gauge fixing. However, also in view of the above considerations, the discussion of the memory effect and its relation to the symmetry acting at Coulombic order remain unaltered.

\section{Phase memory}

Let us consider a pair of electric charges $q$ and $-q$ that are pinned in the positions $\left(r, \mathbf{n}_{1}\right)$ and $\left(r, \mathbf{n}_{2}\right)$ for large $r$. We will now derive the expression for an imprint that the passage of a radiation train leaves on the properties of these particles that is encoded in the phase of their states. What follows is inspired by the four-dimensional discussion of [17]. For a quantum treatment of electromagnetic kick memory see instead [14].

To this purpose, let us assume that, as in the previous section, radiation impinges on the charges only during the interval between two given retarded times $u_{0}$ and $u_{1}$. As we have seen, this means that

$$
\left.\mathcal{A}_{i}\right|_{u_{1}}-\left.\mathcal{A}_{i}\right|_{u_{0}}=\frac{1}{r^{D-4}} \partial_{i} g+\mathcal{O}\left(r^{3-D}\right)
$$


for a suitable angular function $g(\mathbf{n})$. We will assume for simplicity that the gauge field before the onset of radiation is the trivial one.

Let $\left|\psi_{1}\right\rangle=|q\rangle$ and $\left|\psi_{2}\right\rangle=|-q\rangle$ be the initial states in which the charged particles are prepared, which are uniquely labeled by their charges since translational degrees of freedom have been suppressed. Before $u_{0}$, the state $\left|\psi_{2}, \mathbf{n}_{1}\right\rangle$ obtained by the parallel transport of the second state $\left|\psi_{2}\right\rangle$ to the position $\mathbf{n}_{1}$ of the first the charge is

$$
\left|\psi_{2}, \mathbf{n}_{1}\right\rangle=\left|\psi_{2}\right\rangle,
$$

because $\mathcal{A}_{\mu}=0$, so that the corresponding tensor state evaluated in $\mathbf{n}_{1}$ is given by

$$
\left|\psi_{1}, \mathbf{n}_{1}\right\rangle \otimes\left|\psi_{2}, \mathbf{n}_{1}\right\rangle=\left|\psi_{1}\right\rangle \otimes\left|\psi_{2}\right\rangle .
$$

At any fixed $u \geq u_{1}$, instead, the same operation of parallel transport must be performed by calculating

$$
\begin{aligned}
\left|\psi_{2}, \mathbf{n}_{1}\right\rangle & =\exp \left[i q \int_{\mathbf{n}_{2}}^{\mathbf{n}_{1}} \mathcal{A}_{i} d x^{i}\right]\left|\psi_{2}\right\rangle \\
& =\exp \left[i q \frac{g\left(\mathbf{n}_{1}\right)-g\left(\mathbf{n}_{2}\right)}{r^{D-4}}\right]\left|\psi_{2}\right\rangle+\mathcal{O}\left(r^{3-D}\right),
\end{aligned}
$$

where we have employed (3.42). Therefore, after the passage of radiation,

$$
\begin{aligned}
& \left|\psi_{1}, \mathbf{n}_{1}\right\rangle \otimes\left|\psi_{2}, \mathbf{n}_{1}\right\rangle \\
& \quad=\exp \left[i q \frac{g\left(\mathbf{n}_{1}\right)-g\left(\mathbf{n}_{2}\right)}{r^{D-4}}\right]\left|\psi_{1}\right\rangle \otimes\left|\psi_{2}\right\rangle+\mathcal{O}\left(r^{3-D}\right),
\end{aligned}
$$

which displays how the transition between two different radiative vacua, already experimentally detectable by the occurrence of a nontrivial velocity kick for a test charge, is also signaled by the variation of the relative phases in the states obtained by parallel transport of charged particles. Such a phase can be nontrivial provided that the function $g$ is nonconstant, namely when there is a nontrivial memory kick (3.38).
A point that should be stressed is that, in this setup, the states $\left|\psi_{1}\right\rangle$ and $\left|\psi_{2}\right\rangle$ do not evolve, since each particle is kept fixed in its position (its translational quantum numbers are frozen) while electromagnetic radiation cannot change its charge. The relative phase difference occurs entirely as an effect of the evolution of $\mathcal{A}_{\mu}$, which undergoes a transition between two underlying radiative vacua. As we shall see in the following, this aspect is qualitatively different in a nonAbelian theory such as Yang-Mills, where radiation can alter the color charge.

\section{YANG-MILLS MEMORY}

In this section we extend the previous analysis to the non-Abelian case.

\section{A. Yang-Mills theory in the Lorenz gauge}

We consider pure Yang-Mills theory of an antiHermitian gauge field $\mathcal{A}_{\mu}(x)=\mathcal{A}_{\mu}^{A}(x) T^{A}$, where $T^{A}$ are the generators of an $s u(N)$ algebra. The field strength reads $\mathcal{F}_{\mu \nu}=\partial_{\mu} \mathcal{A}_{\nu}-\partial_{\nu} \mathcal{A}_{\mu}+\left[\mathcal{A}_{\mu}, \mathcal{A}_{\nu}\right]$, while the infinitesimal form of the gauge transformation is expressed by $\delta_{\epsilon} \mathcal{A}_{\mu}=\partial_{\mu} \epsilon+$ $\left[\mathcal{A}_{\mu}, \epsilon\right]$ in terms of the gauge parameter $\epsilon(x)=\epsilon^{A}(x) T^{A}$.

We impose the Lorenz gauge condition $\nabla^{\mu} \mathcal{A}_{\mu}=0$, which leaves as residual gauge parameters those that satisfy $\square \epsilon+\left[\mathcal{A}_{\mu}, \partial^{\mu} \epsilon\right]=0$. Furthermore, the equations of motion reduce to $\square \mathcal{A}_{\nu}+\left[\mathcal{A}^{\mu}, \nabla_{\mu} A_{\nu}+\mathcal{F}_{\mu \nu}\right]=0$.

Adopting retarded Bondi coordinates, the Lorenz gauge condition is tantamount to

$$
\partial_{u} \mathcal{A}_{r}=\left(\partial_{r}+\frac{D-2}{r}\right)\left(\mathcal{A}_{r}-\mathcal{A}_{u}\right)+\frac{1}{r^{2}} \mathcal{D} \cdot \mathcal{A},
$$

while the constraint on residual transformations is

$$
\begin{aligned}
& \left(\partial_{u}^{2}-2 \partial_{u} \partial_{r}+\frac{1}{r^{2}} \Delta\right) \epsilon+\frac{D-2}{r}\left(\partial_{r}-\partial_{u}\right) \epsilon \\
& \quad=\left[\partial_{r} \epsilon, \mathcal{A}_{r}-\mathcal{A}_{u}\right]-\left[\partial_{u} \epsilon, \mathcal{A}_{r}\right]+\frac{1}{r^{2}} \gamma^{i j}\left[\mathcal{D}_{i} \epsilon, \mathcal{A}_{j}\right] .
\end{aligned}
$$

The equations of motion are instead

$$
\begin{aligned}
& {\left[\partial_{r}^{2}-2 \partial_{u} \partial_{r}-\frac{D-2}{r}\left(\partial_{u}-\partial_{r}\right)+\frac{1}{r^{2}} \Delta\right] \mathcal{A}_{u}=\left[\mathcal{A}_{u}-\mathcal{A}_{r}, \partial_{r} \mathcal{A}_{u}+\mathcal{F}_{r u}\right]+\left[\mathcal{A}_{r}, \partial_{u} \mathcal{A}_{u}\right]-\frac{\gamma^{i j}}{r^{2}}\left[\mathcal{A}_{i}, \mathcal{D}_{j} \mathcal{A}_{u}+\mathcal{F}_{j u}\right]} \\
& {\left[\partial_{r}^{2}-2 \partial_{u} \partial_{r}-\frac{D-2}{r}\left(\partial_{u}-\partial_{r}\right)+\frac{1}{r^{2}} \Delta\right] \mathcal{A}_{r}+\frac{D-2}{r^{2}}\left(\mathcal{A}_{u}-\mathcal{A}_{r}\right)-\frac{2}{r^{3}} \mathcal{D} \cdot \mathcal{A}} \\
& \quad=\left[\mathcal{A}_{u}-\mathcal{A}_{r}, \partial_{r} \mathcal{A}_{r}\right]+\left[\mathcal{A}_{r}, \partial_{u} \mathcal{A}_{r}+\mathcal{F}_{u r}\right]-\frac{\gamma^{i j}}{r^{2}}\left[\mathcal{A}_{i}, \mathcal{D}_{j} \mathcal{A}_{r}+\mathcal{F}_{j r}\right] \\
& {\left[\left(\partial_{r}^{2}-2 \partial_{u} \partial_{r}+\frac{1}{r^{2}} \Delta\right)-\frac{D-4}{r}\left(\partial_{u}-\partial_{r}\right)-\frac{D-3}{r^{2}}\right] \mathcal{A}_{i}-\frac{2}{r} \mathcal{D}_{i}\left(\mathcal{A}_{u}-\mathcal{A}_{r}\right)} \\
& \quad=\left[\mathcal{A}_{u}-\mathcal{A}_{r},\left(\partial_{r}-\frac{2}{r}\right) \mathcal{A}_{i}+\mathcal{F}_{r i}\right]+\left[\mathcal{A}_{r}, \partial_{u} \mathcal{A}_{i}+\mathcal{F}_{u i}\right]-\frac{\gamma^{j k}}{r^{2}}\left[\mathcal{A}_{j}, \mathcal{D}_{k} \mathcal{A}_{i}+\mathcal{F}_{k i}\right] .
\end{aligned}
$$




\section{B. Asymptotic expansion and color flux}

Performing the usual asymptotic expansion in inverse powers of the radial coordinate $r$, one obtains the following set of equations. Equations (4.1) and (4.2) give

$\partial_{u} A_{r}^{(k+1)}=(D-k-2)\left(A_{r}^{(k)}-A_{u}^{(k)}\right)+\mathcal{D} \cdot A^{(k-1)}$

and

$$
\begin{aligned}
(D- & 2 k-2) \partial_{u} \epsilon^{(k)}-[\Delta-(k-1)(D-k-2)] \epsilon^{(k-1)} \\
= & \sum_{l+m=k}\left(-l\left[\epsilon^{(l)}, A_{u}^{(m)}-A_{r}^{(m)}\right]+\left[\partial_{u} \epsilon^{(l+1)}, A_{r}^{(m)}\right]\right. \\
& \left.\quad-\gamma^{i j}\left[\mathcal{D}_{i} \epsilon^{(l-1)}, A_{j}^{(m)}\right]\right)
\end{aligned}
$$

respectively, while from (4.3) one obtains

$$
\begin{aligned}
(D- & 2 k-2) \partial_{u} A_{u}^{(k)}-[\Delta-(D-k-2)(k-1)] A_{u}^{(k-1)} \\
= & \sum_{l+m=k}\left(\left[A_{r}^{(m)}-A_{u}^{(m)},-l A_{u}^{(l)}+F_{r u}^{(l+1)}\right]-\left[A_{r}^{(m)}, \partial_{u} A_{u}^{(l+1)}\right]\right. \\
& \left.+\gamma^{i j}\left[A_{i}^{(m)}, \mathcal{D}_{j} A_{u}^{(l-1)}+F_{j u}^{(l-1)}\right]\right), \\
(D- & 2 k-2) \partial_{u} A_{r}^{(k)}-[\Delta-k(D-k-1)] A_{r}^{(k-1)} \\
- & (D-2) A_{u}^{(k-1)}+2 \mathcal{D} \cdot A^{(k-2)} \\
= & \sum_{l+m=k}\left(\left[A_{r}^{(m)}-A_{u}^{(m)},-l A_{r}^{(l)}\right]-\left[A_{r}^{(m)}, \partial_{u} A_{r}^{(l+1)}+F_{u r}^{(l+1)}\right]\right. \\
& \left.+\gamma^{i j}\left[A_{i}^{(m)}, \mathcal{D}_{j} A_{r}^{(l-1)}+F_{j r}^{(l-1)}\right]\right), \\
(D- & 2 k-4) \partial_{u} A_{i}^{(k)}-[\Delta-k(D-k-3)-1] A_{i}^{(k-1)} \\
+ & 2 \mathcal{D}_{i}\left(A_{u}^{(k)}-A_{r}^{(k)}\right) \\
= & \sum_{l+m=k}\left(\left[A_{r}^{(m)}-A_{u}^{(m)},-(l+2) A_{i}^{(l)}+F_{r i}^{(l+1)}\right]\right. \\
- & {\left.\left[A_{r}^{(m)}, \partial_{u} A_{i}^{(l+1)}+F_{u i}^{(l+1)}\right]+\gamma^{j k}\left[A_{j}^{(m)}, \mathcal{D}_{k} A_{i}^{(l-1)}+F_{k i}^{(l-1)}\right]\right), }
\end{aligned}
$$

for the corresponding components of the equations of motion. Equations (4.4)-(4.8) appear to order $r^{-(k+1)}$ in the asymptotic expansions.

We choose to adopt the same "radiation" falloff conditions (3.10) that we imposed in the linearized theory: the asymptotic expansions of $\mathcal{A}_{u}$ and $\mathcal{A}_{r}$ start at order $r^{-(D-2) / 2}$ and that of $\mathcal{A}_{i}$ starts at order $r^{-(D-4) / 2}$.

For completeness, and as a cross-check of the choice of falloffs, let us now discuss the definition of the total color charge $\mathcal{Q}(u)=\mathcal{Q}^{A}(u) T^{A}$ and its dependence on the retarded time $u$. In the Lorenz gauge, this quantity is given by the surface integral

$$
\begin{aligned}
\mathcal{Q}^{A}(u)= & \int_{S_{u}} F_{u r}^{A} r^{D-2} d \Omega \\
= & \sum_{k} r^{D-2-k} \int_{S_{u}}\left(\partial_{u} A_{r}^{(k)}+(k-1) A_{u}^{(k-1)}\right. \\
& \left.+\sum_{l+m=k}\left[A_{u}^{(m)}, A_{r}^{(l)}\right]\right)^{A} d \Omega,
\end{aligned}
$$

in the limit $r \rightarrow \infty$. Combining the Lorenz condition (4.4) and the $r$ component of the equations of motion (4.7), one obtains

$$
\begin{aligned}
& (D-2-k)\left(\partial_{u} A_{r}^{(k)}+(k-1) A_{u}^{(k)}\right) \\
& -\mathcal{D}^{i}\left(\mathcal{D}_{i} A_{r}^{(k-1)}+(k-2) A_{i}^{(k-2)}\right) \\
& =\sum_{l+m=k}\left(\left[A_{r}^{(m)}-A_{u}^{(m)},-l A_{r}^{(l)}\right]-\left[A_{r}^{(m)}, \partial_{u} A_{r}^{(l+1)}+F_{u r}^{(l+1)}\right]\right. \\
& \left.\quad+\gamma^{i j}\left[A_{i}^{(m)}, D_{j} A_{r}^{(l-1)}+F_{j r}^{(l-1)}\right]\right) .
\end{aligned}
$$

We see that (4.4) implies $\partial_{u} A_{r}^{\left(\frac{D-2}{2}\right)}=0$ and that (4.10) reduces to

$$
\begin{gathered}
(D-2-k)\left(\partial_{u} A_{r}^{(k)}+(k-1) A_{u}^{(k-1)}\right) \\
=\mathcal{D}^{i}\left(\mathcal{D}_{i} A_{r}^{(k-1)}+(k-2) A_{i}^{(k-2)}\right)
\end{gathered}
$$

for $k<D-2$. The final expression for the color charge may be cast in the form

$$
\mathcal{Q}^{A}(u)=\int_{S_{u}}\left(A_{r}^{(D-3)}+(D-4) A_{u}^{(D-3)}+\left[A_{u}^{\left(\frac{D-2}{2}\right)}, A_{r}^{\left(\frac{D-2}{2}\right)}\right]\right)^{A} d \Omega,
$$

by means of the Lorenz condition (4.4).

Concerning the dependence of $\mathcal{Q}^{A}$ on retarded time, recalling that $\partial_{u} A_{r}^{\left(\frac{D-2}{2}\right)}=0$ by the Lorenz condition, and employing (4.6) and (4.10) for $k=D-3$, we get

$$
\frac{d}{d u} \mathcal{Q}^{A}(u)=\int_{S_{u}} \gamma^{i j}\left[A_{i}^{\left(\frac{D-4}{2}\right)}, \partial_{u} A_{j}^{\left(\frac{D-4}{2}\right)}\right] d \Omega .
$$

This formula provides the flux of the total color charge due to nonlinearities of the theory. Note in particular that the right-hand side involves the radiation components, representing the flux of classical gluons across null infinity.

\section{Yang-Mills memory}

Starting from the radiation falloffs (3.10), we may employ the residual gauge symmetry of the theory to perform a further, recursive gauge fixing. Actually, since the nonlinear corrections to Eq. (3.19) appear to order $q=D-3$ or higher, the discussion of this gauge fixing proceeds as in Sec. III B 1 up to the next to last step. Indeed, 
in accordance with the fact that Yang-Mills theory must encompass both ordinary/linear and null/nonlinear memory, the gauge fixing stops at $q=D-4$ and cannot be performed up until $q=D-3$, as already observed in the case of null electromagnetic memory.

The resulting falloffs after this procedure are thus

$$
\begin{aligned}
\mathcal{A}_{u} & =\sum_{k=D-3}^{\infty} A_{u}^{(k)} r^{-k}, \quad \mathcal{A}_{r}=\sum_{k=\frac{D-2}{2}}^{\infty} A_{r}^{(k)} r^{-k}, \\
\mathcal{A}_{i} & =\sum_{k=\frac{D-4}{2}}^{\infty} A_{i}^{(k)} r^{-k}
\end{aligned}
$$

with residual symmetry parameter

$$
\epsilon\left(u, r, x^{i}\right)=c^{A} T^{A}+\epsilon^{(D-4)}\left(x^{i}\right) r^{4-D}+\cdots,
$$

where $c^{A}$ are constant coefficients.

A colored test particle with charge $Q=Q^{A} T^{A}$ interacts with the background Yang-Mills field by the Wong equations [39]

$$
\dot{P}^{\mu}=\operatorname{tr}\left(Q \mathcal{F}^{\mu \nu}\right) \dot{x}_{\nu}, \quad \dot{Q}=-\dot{x}^{\mu}\left[\mathcal{A}_{\mu}, Q\right] .
$$

Focusing on the region near null infinity, a test "quark," initially at rest, invested by radiation between the retarded times $u_{0}$ and $u_{1}$, will therefore experience the following leading-order momentum kick:

$$
\begin{aligned}
P_{i}^{\prime}-P_{i} & =\int_{u_{0}}^{u_{1}} \operatorname{tr}\left(Q \mathcal{F}_{i u}\right) d u=-\left.\frac{1}{r^{D-4}} \operatorname{tr}\left(Q A_{i}^{(D-4)}\right)\right|_{u_{1}} \\
& =-\frac{1}{r^{D-4}} \operatorname{tr}\left[Q e^{-\epsilon^{(D-4)}} \partial_{i} e^{\epsilon^{(D-4)}}\right]
\end{aligned}
$$

where we have chosen the vacuum configuration at $u=u_{0}$ to be $\mathcal{A}_{\mu}=0$. On the other hand, the color of the test quark will change to leading order, according to

$Q^{\prime}-Q=-\int_{u_{0}}^{u_{1}}\left[\mathcal{A}_{u}, Q\right] d u=-\frac{1}{r^{D-3}} \int_{u_{0}}^{u_{1}}\left[A_{u}^{(D-3)}, Q\right] d u$,

where the $u$ dependence of $\mathcal{A}_{u}$ is governed, according to Eq. (4.7) with $k=D-3$, by

$$
\partial_{u} A_{u}^{(D-3)}=\frac{1}{D-4} \gamma^{i j}\left[A_{i}^{\left(\frac{D-4}{2}\right)}, \partial_{u} A_{j}^{\left(\frac{D-4}{2}\right)}\right] .
$$

Equation (4.19) characterizes the evolution of the charge of the quark in terms of the leading outgoing radiation terms.

In order to better understand the dependence of this momentum kick and of color evolution on the incoming radiation near null infinity, let us further analyze the equations of motion. Combining Eqs. (4.4) and (4.7) we see that

$$
\partial_{u} A_{r}^{(k+1)}=\mathscr{D}_{k} A_{r}^{(k)}
$$

for $k<D-3$, where

$$
\mathscr{D}_{k}=[\Delta+(k-1)(k-D+2)] /(D-2 k-2)
$$

and

$$
\partial_{u} A_{r}^{(D-2)}=\mathscr{D}_{D-3} A_{r}^{(D-3)}-A_{u}^{(D-3)}-\frac{1}{D-4} \mathcal{J}
$$

for $k=D-3$ in dimensions $D>4$, where we have defined

$$
\mathcal{J}=2 \gamma^{i j}\left[A_{i}^{\left(\frac{D-4}{2}\right)}, \mathcal{D}_{j} A_{r}^{\left(\frac{D-2}{2}\right)}\right]-2\left[A_{r}^{\left(\frac{D-2}{2}\right)}, \partial_{u} A_{r}^{\left(\frac{D}{2}\right)}\right] .
$$

Equations (4.4) and (4.7), evaluated for $k=D-3$ and $k=D-2$, respectively, actually imply the following constraint on $\mathcal{J}$ :

$$
\mathcal{J}=-\mathcal{D}^{i}\left[\mathcal{D}_{i} A_{r}^{(D-3)}+(D-4) A_{i}^{(D-4)}\right],
$$

and, taking the derivative of the previous equation with respect to $u$,

$$
\begin{aligned}
& (D-4) \partial_{u} \mathcal{D} \cdot A^{(D-4)} \\
& =-\Delta \partial_{u} A_{r}^{(D-3)}+2\left[A_{r}^{\left(\frac{D-2}{2}\right)}, \partial_{u}^{2} A_{r}^{\left(\frac{D}{2}\right)}\right]-2 \gamma^{i j}\left[\partial_{u} A_{i}^{\left(\frac{D-4}{2}\right)}, \mathcal{D}_{j} A_{r}^{\left(\frac{D-2}{2}\right)}\right] .
\end{aligned}
$$

Starting from Eq. (4.22) and employing (4.20) recursively, we find that

$\partial_{u}^{\left(\frac{D}{2}\right)} A_{r}^{(D-2)}=\prod_{l=\frac{D-2}{2}}^{D-3} \mathscr{D}_{l} \partial_{u} A_{r}^{\left(\frac{D-2}{2}\right)}-\partial_{u}^{\frac{D-2}{2}} A_{u}^{(D-3)}-\frac{1}{D-4} \partial_{u}^{\frac{D-2}{2}} \mathcal{J}$,

and, by Eq. (4.24),

$$
\begin{aligned}
\partial_{u}^{\frac{D-2}{2}} \mathcal{D} \cdot A^{(D-4)}= & \partial_{u}^{\frac{D-2}{2}}\left(\partial_{u} A_{r}^{(D-2)}+A_{u}^{(D-3)}\right) \\
& -\frac{1}{D-4} \partial_{u}^{\frac{D-2}{2}} \Delta A_{r}^{(D-3)}-\prod_{l=\frac{D-2}{2}}^{D-3} \mathscr{D}_{l} \partial_{u} A_{r}^{\left(\frac{D-2}{2}\right)}
\end{aligned}
$$

Equations (4.25) and (4.27) encode the dependence of $\mathcal{D} \cdot A^{(D-4)}$, and hence of the memory kick, on the radiation data encoded in this case by $A_{r}^{\left(\frac{D-2}{2}\right)}$.

In dimension $D=4$, considering again the radiation falloffs (3.10) and an asymptotic series expansion in powers of $1 / r$, the above equations take instead the form 


$$
\begin{gathered}
\mathcal{D}^{i} \partial_{u}\left[A_{i}^{(0)}, A_{r}^{(1)}\right]+\gamma^{i j}\left[A_{i}^{(0)}, \partial_{u}\left[A_{j}^{(0)}, A_{r}^{(1)}\right]\right]=0, \\
\partial_{u} \mathcal{D} \cdot A^{(0)}=\partial_{u}\left(\partial_{u} A_{r}^{(2)}+A_{u}^{(1)}\right) .
\end{gathered}
$$

These coupled equations encode the dependence of $A_{i}^{(0)}$ on the leading electric field and on nonlinear source terms, compatibly with the analysis given in [18]. ${ }^{6}$

\section{Color memory}

Let us now consider a pair of color charges that are pinned in the positions $\left(r, \mathbf{n}_{1}\right)$ and $\left(r, \mathbf{n}_{2}\right)$ for large $r$. Analyzing the effect of the passage of Yang-Mills radiation will provide the non-Abelian analogue of the phase memory effect highlighted in Sec. III D, namely a relative rotation between their states in color space. The content of this section is basically inspired by [18], where color memory was first discussed and whose four-dimensional results we extend to arbitrary even-dimensional spaces.

We consider colored particles in the fundamental representation of the gauge group that, for definiteness, we first take to be $S U(2)$. Assuming that radiation is nontrivial only between two given retarded times $u_{0}$ and $u_{1}$, as we have seen, the angular components of the gauge field satisfy

$$
\left.\mathcal{A}_{i}\right|_{u_{1}}-\left.\mathcal{A}_{i}\right|_{u_{0}}=\frac{1}{r^{D-4}} e^{-\alpha} \partial_{i} e^{\alpha}+\mathcal{O}\left(r^{3-D}\right)
$$

for a suitable $\alpha(\mathbf{n})=\alpha^{A}(\mathbf{n}) T^{A}$, where $T^{A}$ are the $\operatorname{su}(2)$ generators. We have assumed for simplicity that the pure gauge configuration before $u_{0}$ is the trivial one.

Let $\left|\psi_{1}\right\rangle$ and $\left|\psi_{2}\right\rangle$ be the initial states in which the colored particles are prepared. Before $u_{0}$, the state $\left|\psi_{2}, \mathbf{n}_{1}\right\rangle$ that results from the parallel transport of the second state $\left|\psi_{2}\right\rangle$ to the position $\mathbf{n}_{1}$ of the first charge is

$$
\left|\psi_{2}, \mathbf{n}_{1}\right\rangle=\left|\psi_{2}\right\rangle
$$

because $\mathcal{A}_{\mu}=0$. In particular, one can take it to be one of the eigenstates of $T^{3}$, i.e., $|+\rangle$ or $|-\rangle$. In order to build a color singlet state in $\mathbf{n}_{1}$, it then suffices to prepare the superposition

$$
\begin{gathered}
\frac{\left|+, \mathbf{n}_{1}\right\rangle \otimes\left|-, \mathbf{n}_{1}\right\rangle-\left|-, \mathbf{n}_{1}\right\rangle \otimes\left|+, \mathbf{n}_{1}\right\rangle}{\sqrt{2}} \\
=\frac{|+\rangle \otimes|-\rangle-|-\rangle \otimes|+\rangle}{\sqrt{2}} .
\end{gathered}
$$

\footnotetext{
${ }^{6}$ Let us notice that, in order to unravel the symmetry origin of the above effect in $D=4$, one cannot straightforwardly rely on the structure of asymptotic symmetries described in (4.15). It is conceivable that weaker falloff conditions are needed, including $\log$ terms, in the spirit of Sec. V.
}

We remark that, in order for the singlet to be well-defined when $S U(2)$ transformations can depend on the position, it is crucial to build a singlet out of states that have been parallel transported to the same point.

A given state $|\psi\rangle$ evolves according to the covariant conservation equation $[40]^{7}$

$$
\partial_{u}|\psi\rangle=-\mathcal{A}_{u}|\psi\rangle
$$

where we recall that $\mathcal{A}_{u}=\mathcal{O}\left(r^{3-D}\right)$ for large $r$. Thus, the state after the passage of radiation differs with respect to its initial value by

$$
\left.|\psi\rangle\right|_{u_{1}}-\left.|\psi\rangle\right|_{u_{0}}=\mathcal{O}\left(r^{3-D}\right) .
$$

Now, at $u_{1}$ one must also take into account the effect of parallel transport from $\mathbf{n}_{2}$ to $\mathbf{n}_{1}$ on the sphere, given by

$$
\begin{aligned}
\left|\psi_{2}, \mathbf{n}_{1}\right\rangle & =\mathcal{P} \exp \left[-\int_{\mathbf{n}_{2}}^{\mathbf{n}_{1}} \mathcal{A}_{i} d x^{i}\right]\left|\psi_{2}\right\rangle \\
& =\left|\psi_{2}\right\rangle-\frac{1}{r^{D-4}} \int_{\mathbf{n}_{2}}^{\mathbf{n}_{1}} e^{-\alpha} \partial_{i} e^{\alpha} d x^{i}\left|\psi_{2}\right\rangle+\mathcal{O}\left(r^{3-D}\right),
\end{aligned}
$$

where $\mathcal{P}$ denotes path ordering and we have employed (4.30) to establish the second equality. We conclude that the actual state after radiation has passed is no longer the singlet (4.32), but rather

$$
\begin{aligned}
\frac{\left|+, \mathbf{n}_{1}\right\rangle \otimes\left|-, \mathbf{n}_{1}\right\rangle-\left|-, \mathbf{n}_{1}\right\rangle \otimes\left|+, \mathbf{n}_{1}\right\rangle}{\sqrt{2}} & \frac{|+\rangle \otimes|-\rangle-|-\rangle \otimes|+\rangle}{\sqrt{2}} \\
& -\frac{1}{r^{D-4} \sqrt{2}}\left[|+\rangle \otimes \int_{\mathbf{n}_{2}}^{\mathbf{n}_{1}} e^{-\alpha} \partial_{i} e^{\alpha} d x^{i}|-\rangle\right. \\
& \left.-|-\rangle \otimes \int_{\mathbf{n}_{2}}^{\mathbf{n}_{1}} e^{-\alpha} \partial_{i} e^{\alpha} d x^{i}|+\rangle\right]+\mathcal{O}\left(r^{3-D}\right) .
\end{aligned}
$$

A comparison between (4.32) and (4.36) shows that the interaction of the color charges with the external YangMills radiation induces a rotation of the initial state that manifests itself to order $\mathcal{O}\left(r^{4-D}\right)$ in the final state. In other words, a pair of particles initially prepared in a singlet will no longer be in a singlet after the passage of radiation. This color memory can be nontrivial provided that the function $\alpha$ is nonconstant, namely whenever there is also potentially a nontrivial memory kick (3.38).

\footnotetext{
${ }^{7}$ The covariant conservation equation for the color states, which in general reads $\left(\frac{d}{d \tau}+\dot{x}^{\mu} \mathcal{A}_{\mu}\right)|\psi\rangle=0$, implies the Wong equation (4.16) for the color charge $\frac{d}{d \tau} Q+\left[\dot{x}^{\mu} \mathcal{A}_{\mu}, Q\right]=0$, where $Q=Q^{A} T^{A}$ and $Q^{A}=\left\langle\psi\left|T^{A}\right| \psi\right\rangle$.
} 
From a technical point of view, it should be noted how the effect of radiation on each color state is to induce a time evolution to order $\mathcal{O}\left(r^{3-D}\right)$, according to (4.34), which allows one to disentangle it from the leading effect due to the vacuum transition (4.30) undergone by $\mathcal{A}_{i}$, which enters (4.36) to order $\mathcal{O}\left(r^{4-D}\right)$.

A very similar derivation allows one to extend the result to more general states and gauge groups. Adopting an orthonormal basis $|n\rangle$ for the fundamental representation of $S U(N)$, we can consider the superposition

$$
\sum_{n, m} c_{n, m}|n\rangle \otimes|m\rangle
$$

with $\sum_{n, m}\left|c_{n, m}\right|^{2}=1$, prepared before $u_{0}$. Time evolution will induce modifications of $|n\rangle$ that appear to order $\mathcal{O}\left(r^{3-D}\right)$ by $\mathcal{A}_{u}=\mathcal{O}\left(r^{3-D}\right)$. On the other hand, the effect of parallel transport from $\mathbf{n}_{2}$ to $\mathbf{n}_{1}$ performed after $u_{1}$ gives rise to the leading-order correction

$$
-\frac{1}{r^{D-4}} \sum_{n, m} c_{n, m}|n\rangle \otimes \int_{\mathbf{n}_{2}}^{\mathbf{n}_{1}} e^{-\alpha} \partial_{i} e^{\alpha} d x^{i}|m\rangle
$$

to (4.37), due to the nontrivial configuration (4.30) attained by $\mathcal{A}_{i}$.

\section{MORE ON ASYMPTOTIC SYMMETRIES FOR THE MAXWELL THEORY}

So far, we focused on the symmetries directly related to memory effects. As we saw, these symmetries act at Coulombic order so that the corresponding charge (3.22) displays a falloff $\mathcal{O}\left(r^{4-D}\right)$. Thus, they do not comprise asymptotic symmetries, stricto sensu, since the associated charges vanish at $\mathcal{I}^{+}{ }^{8}$ In this section we aim to complement our discussion by performing a more general analysis of the asymptotic symmetry algebra for the Maxwell theory in higher dimensions. ${ }^{9}$

In the radial gauge, in $D=4$, the standard powerlike asymptotic expansion easily allows one to identify the existence of infinite-dimensional asymptotic symmetries. The same result, in the same gauge, can be obtained in higher dimensions upon choosing a powerlike ansatz with leading-order terms as weak as $1 / r$ [24]. (Showing the consistency of this choice in the gravitational case allowed us to identify the Bondi-Metzner-van der Burg-Sachs (BMS) group as the relevant group of asymptotic symmetries for

\footnotetext{
${ }^{8}$ In addition, one should check whether the symmetry is actually canonically generated by those charges. This type of analysis, however, lies beyond the scope of our work.

${ }^{9}$ Let us mention, however, that contrary to the calculation of memory effects, which appear to be tightly related to the properties of the wave equation in even-dimensional spacetimes, these further investigations of asymptotic symmetries admit an extension to spacetimes of not only even but also odd dimensions. While focusing on the former case in the main discussion, we will comment along the way on the differences arising in the latter.
}

asymptotically flat spacetimes of arbitrary even dimensions [41].) In this section we perform a similar analysis in the Lorenz gauge, highlighting the existence of full-fledged, infinite-dimensional asymptotic symmetries in any $D$, while also proposing a procedure for computing finite and nonvanishing associated charges on $\mathcal{I}$. While in agreement with dedicated literature concerning the gravitational case [16,41], the actual interplay between these symmetries with finite charges and those responsible for memory effects, whose charges, differently, vanish on $\mathcal{I}$, probably deserves further conceptual clarification.

On a more technical side, one first observes that, differently from the radial gauge, in the Lorenz gauge a mere powerlike ansatz with leading-order falloffs as weak as $1 / r$ does not allow one to recover a nontrivial structure for the asymptotic symmetry group. In order to display the latter, one finds the need for generalizing the asymptotic expansion by allowing for logarithmic terms. In this section we will provide an explicit realization to this effect, while also discussing the nonobvious issue of the finiteness of the corresponding soft surface charges.

The observed difference between radial and Lorenz gauges is one concrete facet of a general issue concerning the possible gauge dependence of the asymptotic analysis [36,42-44]. While in some instances, such as the present one, this problem has been clarified, the general systematics may still deserve further attention.

\section{A. Polyhomogeneous expansion for $D \geq 4$}

In the Lorenz gauge, in order to investigate the possible existence of large gauge symmetries acting at $\mathcal{I}^{+}$we look for solutions to Eq. (3.2) with the boundary condition

$$
\lim _{r \rightarrow \infty} \epsilon(u, r, \mathbf{n})=\epsilon^{(0)}(u, \mathbf{n})
$$

for some nonconstant function $\epsilon^{(0)}(u, \mathbf{n})$. As it turns out, a power-law ansatz

$$
\epsilon=\sum_{k=0}^{\infty} \frac{\epsilon^{(k)}(u, \mathbf{n})}{r^{k}}
$$

effectively selects only the global symmetry in any even dimension $D \geq 4$. Indeed, considering (3.6) with $k=0,1, \ldots, \frac{D-2}{2}$, we have

$$
\begin{aligned}
(D-2) \partial_{u} \epsilon^{(0)} & =0, \\
(D-4) \partial_{u} \epsilon^{(1)} & =\Delta \epsilon^{(0)}, \\
\vdots & \\
2 \partial_{u} \epsilon^{\left(\frac{D-4}{2}\right)} & =\left[\Delta-\frac{D(D-6)}{4}\right] \epsilon^{\left(\frac{D-6}{4}\right)}, \\
0 & =\left[\Delta-\frac{(D-4)(D-2)}{4}\right] \epsilon^{\left(\frac{D-4}{2}\right)} .
\end{aligned}
$$


The last equation sets $\epsilon^{\left(\frac{D-4}{2}\right)}$ to zero for any $D \geq 6$, and to a constant for $D=4$. Then, the above system recursively sets to zero the other components $\epsilon^{(k)}$ all the way up to $\epsilon^{(0)}$ since the corresponding operators are all invertible. Eventually, $\epsilon^{(0)}$ must be a constant by $\partial_{u} \epsilon^{(0)}=0$ and $\Delta \epsilon^{(0)}=0$.

One concludes that the power-law ansatz does not allow for an enhanced asymptotic symmetry sitting at the order $\mathcal{O}(1)$. In order to retrieve it, one needs a more general ansatz. We find that it is sufficient, to this purpose, to consider the following type of asymptotic expansion ${ }^{10}$ involving also a logarithmic dependence on $r$ [30,34-36]:

$\epsilon(u, r, \mathbf{n})=\sum_{k=0}^{\infty} \frac{\epsilon^{(k)}(u, \mathbf{n})}{r^{k}}+\sum_{k=\frac{D-2}{2}}^{\infty} \frac{\lambda^{(k)}(u, \mathbf{n}) \log r}{r^{k}}$.

In this fashion, the last equation in (5.3) becomes modified by the presence of the logarithmic branch and yields

$$
2 \partial_{u} \lambda^{\left(\frac{D-2}{2}\right)}=\left[\Delta-\frac{(D-4)(D-2)}{4}\right] \epsilon^{\left(\frac{D-4}{2}\right)},
$$

an equation that determines the $u$ dependence of $\lambda^{\left(\frac{D-2}{2}\right)}$, thus still allowing for an arbitrary $\epsilon^{(0)}(\mathbf{n})$.

Indeed, the recursion relations expressing the equation $\square \epsilon=0 \mathrm{read}$

$$
\begin{aligned}
& (D-2-2 k) \partial_{u} \epsilon^{(k)}+2 \partial_{u} \lambda^{(k)} \\
& =[\Delta+(k-1)(k-D+2)] \epsilon^{(k-1)}+(D-1-2 k) \lambda^{(k-1)}, \\
& (D-2-2 k) \partial_{u} \lambda^{(k)}=[\Delta+(k-1)(k-D+2)] \lambda^{(k-1)},
\end{aligned}
$$

and hence can be solved by direct integration with respect to $u$. Explicitly, for $k<\frac{D-2}{2}$, they reduce to the familiar system

$$
(D-2-2 k) \partial_{u} \epsilon^{(k)}=[\Delta+(k-1)(k-D+2)] \epsilon^{(k-1)},
$$

so that the solution is given by a polynomial in $u$ with angle-dependent coefficients $C_{j, k}(\mathbf{n})$, with $0 \leq j \leq k$, and is uniquely determined by specifying the integration functions $\hat{\epsilon}^{(k)}(\mathbf{n})$ :

$$
\epsilon^{(k)}(u, \mathbf{n})=\sum_{j=0}^{k} C_{j, k}(\mathbf{n}) u^{j},
$$

with

\footnotetext{
${ }^{10}$ The fully polyhomogeneous ansatz would be of the form $\sum_{k, j} r^{-k}(\log r)^{j} \epsilon^{(k, j)}(u, \mathbf{n})$, involving arbitrary powers of $\log r$. We do not explore this more general possibility in the present paper.
}

$$
C_{j, k}(\mathbf{n})= \begin{cases}\hat{\epsilon}^{(k)}(\mathbf{n}) & \text { if } j=0, \\ \frac{1}{j !} \prod_{l=k-j+1}^{k} \mathscr{D}_{l} \hat{\epsilon}^{k-l}(\mathbf{n}) & \text { otherwise }\end{cases}
$$

where $\mathscr{D}_{l}$ was defined in (4.21). If $k=\frac{D-2}{2}$, Eq. (5.6) reduces to (5.5), so that

$$
\begin{aligned}
\lambda^{\left(\frac{D-2}{2}\right)}(u, \mathbf{n})= & \frac{1}{2} \int_{0}^{u}\left[\Delta-\frac{(D-4)(D-2)}{4}\right] \epsilon^{\left(\frac{D-4}{2}\right)}\left(u^{\prime}, \mathbf{n}\right) d u^{\prime} \\
& +\hat{\lambda}^{\left(\frac{D-2}{2}\right)}(\mathbf{n}),
\end{aligned}
$$

with a suitable integration function, while $\epsilon^{\left(\frac{D-2}{2}\right)}(u, \mathbf{n})$ stays unconstrained. For $k>\frac{D-2}{2}$, we can recast (5.6) in the slightly more suggestive form

$$
\begin{aligned}
(D-2-2 k) \partial_{u} \lambda^{(k)}= & {[\Delta+(k-1)(k-D+2)] \lambda^{(k-1)} } \\
(D-2-2 k) \partial_{u} \epsilon^{(k)}= & {[\Delta+(k-1)(k-D+2)] \epsilon^{(k-1)} } \\
& +\left[\frac{2}{2 k-D+2}(\Delta+(k-1)(k-D+2))\right. \\
& +(D-1-2 k)] \lambda^{(k-1)}
\end{aligned}
$$

In this way, it becomes clear that these two equations can always be solved by first finding the integral of the first equation directly, and then substituting it in the second one where it acts as a "source" term on the right-hand side.

To summarize, a solution of (5.6) is specified by assigning a set of integration functions $\hat{\epsilon}^{(k)}(\mathbf{n})$, for $k \geq 0$, and $\hat{\lambda}^{(k)}(\mathbf{n})$, for $k \geq \frac{D-2}{2}$, together with an arbitrary function $\epsilon^{\left(\frac{D-2}{2}\right)}(u, \mathbf{n})$. In particular, this achieves the boundary condition (5.1) as $r \rightarrow \infty$, where $\epsilon^{(0)}(\mathbf{n})$ is an arbitrary function on the celestial sphere.

The situation is simpler in odd dimensions, where the recursion relation (3.6) can be solved compatibly with the boundary condition (5.1) without the need of introducing logarithmic terms. The technical reason is that, when $D$ is odd, the left-hand side of (3.6) never vanishes for integer $k$.

By means of the transformations

$$
\mathcal{A}_{\mu} \mapsto \mathcal{A}_{\mu}+\partial_{\mu} \epsilon
$$

parametrized by the gauge parameters thus obtained, we can then act on solutions to Maxwell's equations in the Lorenz gauge characterized by the radiation falloffs (3.10) and generate a wider solution space. In particular, in view of the expansion (5.4) of $\epsilon$, this type of solutions will generically exhibit the following asymptotic behavior as $r \rightarrow \infty$ :

$$
\mathcal{A}_{u}=\mathcal{O}\left(r^{-1}\right), \quad \mathcal{A}_{r}=\mathcal{O}\left(r^{-2}\right), \quad \mathcal{A}_{i}=\mathcal{O}\left(r^{0}\right),
$$

in any $D \geq 5$, while 
$\mathcal{A}_{u}=\mathcal{O}\left(r^{-1} \log r\right), \quad \mathcal{A}_{r}=\mathcal{O}\left(r^{-1}\right), \quad \mathcal{A}_{i}=\mathcal{O}\left(r^{0}\right)$,

in dimension $D=4$.

It is important to remark that, since such solutions differ from those characterized by (3.10) by a pure gradient, they will still retain all the corresponding physical properties, such as the finiteness of the energy flux at any given retarded time.

As far as (5.12) alone is concerned, one could in principle consider parameters $\epsilon$ that obey arbitrary asymptotics near $\mathcal{I}^{+}$. However, the ones of interest to us are those that define asymptotic symmetries, i.e., parameters that give rise to finite and nonvanishing asymptotic surface charges. In the next section we shall see that this is indeed the case for our class of solutions identified by (5.1).

\section{B. Asymptotic charges}

As it is usually the case in the presence of radiation, the naive surface charge associated with the symmetry (5.4), namely

$\mathcal{Q}_{\epsilon}(u)=\lim _{r \rightarrow \infty} \oint_{S_{u}} \mathcal{F}_{u r}(u, r, \mathbf{n}) \epsilon(u, r, \mathbf{n}) r^{D-2} d \Omega(\mathbf{n})$,

is formally ill defined, because the right-hand side contains terms of the type, e.g.,

$$
r^{\frac{D-4}{2}} \oint_{S_{u}} F_{u r}^{\left(\frac{D}{2}\right)} \epsilon^{(0)}(\mathbf{n}) d \Omega
$$

which do not vanish, even after imposing the equations of motion, precisely due to the presence of an arbitrary parameter $\epsilon^{(0)}(\mathbf{n})$.

Such difficulties are absent in the case of the global charges because the equations of motion always ensure that these potentially dangerous terms are actually zero. Indeed, $\nabla_{\mu} \mathcal{F}^{\mu \nu}=0$ away from electric charges, so that $\int_{S_{u, r}} \mathcal{F}_{\mu \nu} d x^{\mu \nu}$ cannot depend on $r$, nor in fact on $u$, because it must be independent of the specific $(D-2)$ surface under consideration.

It should be noted, however, that, for the general variation with parameter (5.4), the above difficulties arise only if one attempts to calculate the surface charge by integrating over a sphere at a given retarded time $u$ and radius $r$ and then lets $r$ tend to infinity. On the other hand, the calculation of the charge on a Cauchy surface still gives a well-defined result [36]. For instance, under the simplifying assumption that the electromagnetic field due to radiation vanishes for $u<u_{0}$ in a neighborhood of future null infinity, the calculation of the surface charge indeed yields

$$
\begin{aligned}
\mathcal{Q}_{\epsilon}(u) & =\oint_{S_{u}} F_{u r}^{(D-2)} \epsilon^{(0)} d \Omega \\
& =\oint_{S_{u}}\left[A_{r}^{(D-3)}+(D-4) A_{u}^{(D-3)}\right] \epsilon^{(0)} d \Omega,
\end{aligned}
$$

for fixed $u<u_{0}$, since all radiation components $F_{u r}^{(k)}$, for $k<D-2$, vanish for a stationary solution (see the discussion in Sec. III C) and $S_{u}$ is indeed the boundary of a Cauchy surface. Then letting $u$ approach $-\infty$, one has

$$
\begin{aligned}
\mathcal{Q}_{\epsilon}(-\infty) & =\oint_{\mathcal{I}_{ \pm}^{+}} F_{u r}^{(D-2)} \epsilon^{(0)} d \Omega \\
& =\oint_{\mathcal{I}_{-}^{ \pm}}\left[A_{r}^{(D-3)}+(D-4) A_{u}^{(D-3)}\right] \epsilon^{(0)} d \Omega .
\end{aligned}
$$

For $u<u_{0}$, the quantity $\mathcal{Q}_{\epsilon}(u)$ must match the analogous surface integral calculated at spatial infinity because in both cases the Noether two-form is integrated over the boundary of a Cauchy surface, in view of the requirement that no radiation be present in a neighborhood of $\mathcal{I}^{+}$for $u<u_{0}$.

In force of the considerations above, we now define the charge $Q_{\epsilon}(u)$ for all the values of $u$ as follows. For $u<u_{0}$ we define it as in (5.17), while for $u \geq u_{0}$, even in the presence of radiation, we define it as the evolution of (5.17) under the equations of motion. Indeed, the Maxwell equation $\nabla \cdot \mathcal{F}_{r}=0$ gives

$$
\left(\partial_{r}+\frac{D-2}{r}\right) \mathcal{F}_{u r}=\frac{1}{r^{2}} \mathcal{D} \cdot \mathcal{F}_{r}
$$

while $\nabla \cdot \mathcal{F}_{u}=0$ reads

$$
\begin{aligned}
\partial_{u} \mathcal{F}_{u r} & =\left(\partial_{r}+\frac{D-2}{r}\right) \mathcal{F}_{u r}+\frac{1}{r^{2}} \mathcal{D} \cdot \mathcal{F}_{u} \\
\Rightarrow \partial_{u} \mathcal{F}_{u r} & =\frac{1}{r^{2}} \mathcal{D} \cdot\left(\mathcal{F}_{u}+\mathcal{F}_{r}\right),
\end{aligned}
$$

and hence

$$
\frac{d}{d u} \mathcal{Q}_{\epsilon}(u)=\oint_{S_{u}} \mathcal{D} \cdot\left(F_{u}^{(D-4)}-F_{r}^{(D-4)}\right) \epsilon^{(0)} d \Omega
$$

Analogous considerations allow one to introduce welldefined surface charges evaluated at $\mathcal{I}^{-}$.

From the perspective of the analysis performed in Sec. II, it is possible to explicitly calculate the soft charges according to the above strategy. Restricting to the case of a massive charge in dimension $D=6$ that starts moving at $t=0$, the integral of $\mathcal{F}_{u r} \epsilon^{(0)}$ on a sphere at fixed retarded time $u$ and radius $r$ yields 


$$
\begin{aligned}
\mathcal{Q}_{\epsilon}(r, u)= & r \frac{\delta(u)}{8 \pi^{2}} \oint \frac{\mathbf{n} \cdot \mathbf{v}(3 \mathbf{n} \cdot \mathbf{v}-4)-\mathbf{v}^{2}}{(1-\mathbf{n} \cdot \mathbf{v})^{2}} \epsilon^{(0)}(\mathbf{n}) d \Omega \\
& +\frac{3}{8 \pi^{2}} \oint\left[\theta(-u)+\theta(u) \frac{\left(1-\mathbf{v}^{2}\right)^{2}}{(1-\mathbf{n} \cdot \mathbf{v})^{4}}\right] \epsilon^{(0)}(\mathbf{n}) d \Omega .
\end{aligned}
$$

Except for the case of the electric charge $\epsilon^{(0)}=1$, where the first integral vanishes identically, the limit of this surface charge as $r \rightarrow \infty$ is ill defined in the presence of radiation, namely on the forward light cone $u=0$, due to the linear divergence appearing in the first line. However, the charge is well defined on $\mathcal{I}^{+}$before and after the passage of radiation, $u \neq 0$, and reads

$\mathcal{Q}_{\epsilon}(u)=\frac{3}{8 \pi^{2}} \oint\left[\theta(-u)+\theta(u) \frac{\left(1-\mathbf{v}^{2}\right)^{2}}{(1-\mathbf{n} \cdot \mathbf{v})^{4}}\right] \epsilon^{(0)}(\mathbf{n}) d \Omega$.

For $\epsilon^{(0)}=0$, this quantity reduces to the (constant) electric charge $Q=1$, while for more general parameters $\epsilon^{(0)}$, the soft charge exhibits a jump discontinuity at $u=0$, which measures the fact that the particle is no longer static for $u>0$ in a manner akin to the memory effect itself.

Performing instead the limit $r \rightarrow \infty$ at fixed time $t$, it is also possible to verify the matching between the surface charge evaluated at null infinity before the onset of radiation, for $u<0$ (or, equivalently, at $\mathcal{I}_{-}^{+}$), and the Hamiltonian charge $\mathcal{H}_{\epsilon}(t)$, obtained by integrating on a slice at fixed time $t$. Indeed, taking (B18) into account and writing the result in terms of polar coordinates $t, r$, and $\mathbf{n}$, we have, for the scalar field,

$$
\begin{aligned}
8 \pi^{2} \varphi= & \frac{\delta(t-r)}{\gamma(\mathbf{v})(1-\mathbf{n} \cdot \mathbf{v}) r^{2}}+\frac{\theta(t-r)}{\gamma(\mathbf{v})^{3}(1-\mathbf{n} \cdot \mathbf{v})^{3} r^{3}} \Delta(t-r, r)^{-3 / 2} \\
& -\frac{\delta(t-r)}{r^{2}}+\frac{\theta(r-t)}{r^{3}}
\end{aligned}
$$

The corresponding electromagnetic potential is given by $\mathcal{A}^{\mu}=\left(\mathcal{A}^{0}, \mathbf{A}\right)=-\gamma(\mathbf{v})(1, \mathbf{v}) \varphi$, for $t>r$, and $A^{\mu}=(\varphi, 0)$, for $t<r$. The radial component of the electric field then yields $\mathcal{F}_{t r}=3 r^{-4}$ as $r \rightarrow \infty$ for fixed $t$ and hence

$$
\mathcal{H}_{\epsilon}(t)=\frac{3}{8 \pi^{2}} \oint \epsilon^{(0)}(\mathbf{n}) d \Omega=\mathcal{Q}_{\epsilon}(u<0) .
$$

Similar arguments showing the finiteness of the Hamiltonian charge in higher dimensions have been given, in the case of a linearized spin-two field in retarded Bondi gauge, in [45], while a renormalization procedure has been recently proposed, for the Maxwell theory in the radial gauge, in [46] (for the general definition of surface charges see [47]). The relation between asymptotic charges and the soft photon theorem in any $D$ was recently clarified in
[34,35]. In Appendix D we present the details of the computation for the case of interest in our work.

\section{ACKNOWLEDGMENTS}

We are grateful to Glenn Barnich and Cédric Troessaert for discussions and exchanges. A. C. and D. F. express their gratitude to the Erwin Schrödinger International Institute for Mathematics and Physics (ESI) of Vienna for the kind hospitality extended to them during the preparation of this work, in the occasion of the workshop "Higher spins and holography." The work of A. C. was partially supported by the Fund for Scientific Research-FNRS PDR "Fundamental issues in extended gravity," Grant No. T.0022.19. The work of C. H. was partially supported by the Istituto Nazionale di Fisica Nucleare (Iniziativa Specifica GSS-Pi) and by the Fund for Scientific Research-FNRS, Belgium; he also acknowledges the hospitality of ESI. A. C. and C. H. gratefully acknowledge the University Roma Tre for hospitality.

\section{APPENDIX A: NOTATION}

Retarded Bondi coordinates are a retarded time $u=t-r$, a radial coordinate $r$, and angular coordinates $x^{i}$ on the Euclidean unit $(D-2)$-sphere, with metric $\gamma_{i j}$. The Minkowski metric, in such coordinates, reads

$$
d s^{2}=-d u^{2}-2 d u d r+r^{2} \gamma_{i j} d x^{i} d x^{j},
$$

while the (nonvanishing) Christoffel symbols are

$$
\begin{aligned}
\Gamma_{r j}^{i} & =\frac{1}{r} \delta^{i}, \quad \Gamma^{u}{ }_{i j}=-\Gamma^{r}{ }_{i j}=r \gamma_{i j}, \\
\Gamma^{i}{ }_{j k} & =\frac{1}{2} \gamma^{i l}\left(\partial_{j} \gamma_{l k}+\partial_{k} \gamma_{j l}-\partial_{l} \gamma_{j k}\right) .
\end{aligned}
$$

With $\mathcal{D}_{i}$ we denote the covariant derivative associated with $\gamma_{i j}$ and $\Delta=\mathcal{D}_{i} \mathcal{D}^{i}$ is the corresponding Laplace-Beltrami operator. In particular, the d'Alembert operator $\square$ acting on a scalar $\varphi$ takes the explicit form

$\square \varphi=-\left(2 \partial_{r}+\frac{D-2}{r}\right) \partial_{u} \varphi+\left(\partial_{r}^{2}+\frac{D-2}{r} \partial_{r}+\frac{1}{r^{2}} \Delta\right) \varphi$.

We find it useful to also employ the notation $\mathbf{n}=\mathbf{n}\left(x^{i}\right)$ for unit vectors identifying points on the sphere, in terms of which $\gamma_{i j}=\partial_{i} \mathbf{n} \cdot \partial_{j} \mathbf{n}$.

\section{APPENDIX B: CLASSICAL SCALAR SOLUTIONS AND MEMORY EFFECTS}

In this appendix, we derive explicit solutions of the scalar wave equation with different types of background sources and calculate the associated memory effects. This provides a useful warming-up for our discussion of electromagnetic 
memory effects in the Lorenz gauge in even dimensions, while also allowing us to shed some light on the nature of memory effects in odd dimensions.

\section{Scalar fields in even $D$}

Let us first consider a particle with charge $q$ under the scalar field $\varphi$ that is created in the origin at $t=0$. The field generated by this process is obtained by solving the wave equation

$$
-\square \varphi(t, \mathbf{x})=q \theta(t) \delta(\mathbf{x})
$$

(we adopt the convention $-\square=-\eta^{\mu \nu} \partial_{\mu} \partial_{\nu}=\partial_{t}^{2}-\nabla^{2}$ ). The solution is given by the convolution of the source on the right-hand side with the ( $D$-dimensional) retarded wave propagator $G_{D}^{\text {ret }}(x)$, i.e., in this case

$$
\varphi(t, \mathbf{x})=q \int_{-\infty}^{t} G_{D}^{\mathrm{ret}}(\tau, \mathbf{x}) d \tau .
$$

The field generated by a particle that is destroyed in the origin can then be obtained by time reversal of the above solution, while the field for a moving particle can be calculated by applying a Lorentz boost.

Let us recall that [48], for even $D \geq 2$, the retarded propagator is

$$
G_{D}^{\mathrm{ret}}(x)=\frac{1}{2 \pi^{D / 2-1}} \delta^{\left(\frac{D-4}{2}\right)}\left(x^{2}\right) \theta\left(x^{0}\right),
$$

where $\theta$ is the Heaviside distribution. Restricting to even $D \geq 4$, and using the chain rule for the distribution $\delta^{\left(\frac{D-4}{2}\right)}\left(x^{2}\right)$, the propagator (B3) can be recast as

$$
G_{D}^{\mathrm{ret}}(t, \mathbf{x})=\sum_{k=0}^{D / 2-2} c_{D, k} \frac{\delta^{(D / 2-2-k)}(u)}{r^{D / 2-1+k}},
$$

where $u=t-r$ and $r=|\mathbf{x}|$, while $c_{D, k}$ are the coefficients

$$
c_{D, k}=\frac{1}{2(2 \pi)^{\frac{D}{2}-1}} \frac{\left(\frac{D}{2}-2+k\right) !}{2^{k}\left(\frac{D}{2}-2-k\right) ! k !} .
$$

In particular, note that

$c_{D, 0}=\frac{1}{2(2 \pi)^{\frac{D}{2}-1}}, \quad c_{D, \frac{D}{2}-3}=c_{D, \frac{D}{2}-2}=\frac{1}{(D-3) \Omega_{D-2}}$,

where $\Omega_{D-2}$ is the area of the $(D-2)$-dimensional Euclidean unit sphere. The resulting scalar field is thus

$$
\varphi(u, r)=q \sum_{k=0}^{D / 2-2} c_{D, k} \frac{\theta^{(D / 2-2-k)}(u)}{r^{D / 2-1+k}} .
$$

Notice that only the term associated with $k=D / 2-2$ gives rise to a persistent field for fixed $r$, while the other terms have support localized at $u=0$, namely on the future-directed light cone with vertex at the particle's creation. This is a general consequence of the recursion relation (3.6), namely

$$
(D-2 k-2) \partial_{u} \varphi^{(k)}=[\Delta+(k-1)(k-D+2)] \varphi^{(k-1)},
$$

obeyed by $\varphi=\sum_{k} \varphi^{(k)} r^{-k}$ near future null infinity, which requires $[\Delta+k(k-D+3)] \varphi^{(k)}=0$ for $0<k<D-3$, and hence $\varphi^{(k)}=0$, for any stationary solution.

Now, a test particle with charge $Q$, held in place at a distance $r$ from the origin, will be subject to a force $f_{\mu}=$ $Q \partial_{\mu} \varphi(u, r)$ at a given retarded time $u$ due to the presence of the scalar field. Hence, its $D$-momentum $P_{\mu}$ will in general be subject to the leading-order variation

$$
\left.P_{\mu}\right|_{u}-\left.P_{\mu}\right|_{u=-\infty}=Q \int_{-\infty}^{u} \partial_{\mu} \varphi\left(u^{\prime}, r\right) d u^{\prime} .
$$

For this very simple example, this quantity can be calculated explicitly for any even $D$. The variations of $P_{u}$ and $P_{r}$ in particular yield

$$
\begin{aligned}
\left.P_{u}\right|_{u>0}-\left.P_{u}\right|_{u<0} & =Q \int_{-\infty}^{+\infty} \partial_{u} \varphi\left(u^{\prime}, r\right) d u^{\prime} \\
& =\frac{Q q}{(D-3) \Omega_{D-2} r^{D-3}} \quad \text { for } u>0
\end{aligned}
$$

and

$$
\begin{aligned}
\left.P_{r}\right|_{u>0}-\left.P_{r}\right|_{u<0} & =Q \int_{-\infty}^{+\infty} \partial_{r} \varphi\left(u^{\prime}, r\right) d u^{\prime} \\
& =-\frac{(D-4) Q q}{(D-3) \Omega_{D-2} r^{D-3}} .
\end{aligned}
$$

Equation (B10) simply expresses the fact that the test particle will start feeling the Coulombic interaction energy with the newly created particle in the origin as soon as it crosses the light cone subtended by the origin of spacetime. On the other hand, (B11) tells us that the test particle will feel an instantaneous, radial momentum kick, for even dimensions greater than four. Since this process is spherically symmetric, the variations of the angular components $P_{i}$ vanish identically.

The field emitted by a particle destroyed in the origin at $t=0$ is obtained by sending $u \mapsto-u$ in (B7). The case of a particle moving with velocity $\mathbf{v}$ can instead be obtained by boosting (B7):

$t \mapsto \gamma(\mathbf{v})(t-\mathbf{v} \cdot \mathbf{x}), \quad \mathbf{x} \mapsto \mathbf{x}+\mathbf{v}(\gamma(\mathbf{v})-1) \frac{\mathbf{v} \cdot \mathbf{x}}{\mathbf{v}^{2}}-\gamma(\mathbf{v}) \mathbf{v} t$,

which gives, for large $r$, denoting $\mathbf{n}=\mathbf{x} / r$, 


$$
\begin{aligned}
u & \mapsto u \gamma(\mathbf{v})^{-1}(1-\mathbf{n} \cdot \mathbf{v})^{-1}+\mathcal{O}\left(r^{-1}\right), \\
r & \mapsto r \gamma(\mathbf{v})(1-\mathbf{n} \cdot \mathbf{v})+\mathcal{O}(1) .
\end{aligned}
$$

We can then cast the boosted solution in the following form:

$$
\begin{aligned}
\varphi(u, r, \mathbf{n})= & \frac{q \theta(u)}{(D-3) \Omega_{D-2}[\gamma(\mathbf{v})(1-\mathbf{n} \cdot \mathbf{v}) r]^{D-3}} \\
& +\bar{\varphi}(u, r, \mathbf{n})+\mathcal{O}\left(r^{2-D}\right),
\end{aligned}
$$

where $\bar{\varphi}$ is a sum of terms of the type

$$
f_{\alpha}(r, \mathbf{n}) \delta^{(\alpha)}(u), \quad \text { with } \quad \alpha \geq 0,
$$

namely, whose support is localized on the light cone. Let us stress that the terms in $\bar{\varphi}$ formally dominate the asymptotic expansion of $\varphi$ as $r \rightarrow \infty$. However, these terms will not contribute to the leading $u$ component of the momentum kick due to the presence of $\delta(u)$ and its derivatives. We can therefore conclude that

$$
\begin{aligned}
\left.P_{u}\right|_{u>0}-\left.P_{u}\right|_{u<0} & =Q \int_{-\infty}^{+\infty} \partial_{u} \varphi d u^{\prime} \\
& =\frac{q Q}{(D-3) \Omega_{D-2}[\gamma(\mathbf{v})(1-\mathbf{n} \cdot \mathbf{v}) r]^{D-3}},
\end{aligned}
$$

in any even $D$. This is, not surprisingly, just the analogue of Eq. (B10) for the Coulombic energy in which one needs to account for the relativistic length contraction.

For a more general scattering process involving a number of "in" and "out" particles destroyed or created in the origin, the result is obtained by linearly superposing solutions and therefore reads $\left(\eta_{a}=-1\right.$ for an incoming particle and $\eta_{a}=+1$ for an outgoing one)

$$
\begin{aligned}
& \left.P_{u}\right|_{u>0}-\left.P_{u}\right|_{u<0} \\
& =\sum_{a \in \text { in } / \text { out }} \frac{\eta_{a} q_{a} Q}{(D-3) \Omega_{D-2}\left[\gamma\left(\mathbf{v}_{a}\right)\left(1-\mathbf{n} \cdot \mathbf{v}_{a}\right) r\right]^{D-3}} .
\end{aligned}
$$

Calculating radial and angular components of $P_{\mu}$ requires more effort, since they arise instead from the terms proportional to $\delta(u)$ whose number increases with the spacetime dimension. They have been given for any even dimension in [10] in terms of derivatives of a generating function. For our present, illustrative, purposes, it suffices to consider the first relevant case $D=6$, where the exact solution in the case of the particle created in the origin with velocity $\mathbf{v}$ is given by

$8 \pi^{2} \varphi=\frac{\delta(u)}{\gamma(\mathbf{v})(1-\mathbf{n} \cdot \mathbf{v}) r^{2}}+\frac{\theta(u)}{\gamma(\mathbf{v})^{3}(1-\mathbf{n} \cdot \mathbf{v})^{3} r^{3}} \Delta(u, r)^{-3 / 2}$ with

$$
\Delta(u, r)=1+\frac{2 u\left(\mathbf{v}^{2}-\mathbf{n} \cdot \mathbf{v}\right)}{r(1-\mathbf{n} \cdot \mathbf{v})^{2}}+\frac{u^{2} \mathbf{v}^{2}}{r^{2}(1-\mathbf{n} \cdot \mathbf{v})^{2}} .
$$

The corresponding radial and angular memory effects in $D=6$ are then, to leading order,

$$
\begin{gathered}
\left.P_{r}\right|_{u>0}-\left.P_{r}\right|_{u<0}=\frac{-2 Q q}{8 \pi^{2} \gamma(\mathbf{v})(1-\mathbf{n} \cdot \mathbf{v}) r^{3}}, \\
\left.P_{i}\right|_{u>0}-\left.P_{i}\right|_{u<0}=\frac{v_{i} Q q}{8 \pi^{2} \gamma(\mathbf{v})(1-\mathbf{n} \cdot \mathbf{v}) r^{2}},
\end{gathered}
$$

where $v_{i}=\partial_{i} \mathbf{n} \cdot \mathbf{v}$ is the component of the particle's velocity in the $i$ th angular direction.

While the above examples illustrate the phenomenon of ordinary memory associated with the field emitted to massive charges that move in the bulk of the spacetime, we can also consider the wave equation with a source term characterizing the presence of a massless charged particle, moving along a given direction $\hat{\mathbf{x}}$ :

$$
-\square \varphi=q \delta(\mathbf{x}-\hat{\mathbf{x}} t),
$$

with $|\hat{\mathbf{x}}|=1$. This equation can conveniently be solved for any even $D \geq 6$ by going to retarded coordinates, where it reads

$$
\begin{aligned}
\left(2 \partial_{r}+\frac{D-2}{r}\right) \partial_{u} \varphi= & \left(\partial_{r}^{2}+\frac{D-2}{r} \partial_{r}+\frac{1}{r^{2}} \Delta\right) \varphi \\
& +\frac{q}{r^{D-2}} \delta(u) \delta(\mathbf{n}, \hat{\mathbf{x}})
\end{aligned}
$$

and performing the usual asymptotic expansion $\varphi(u, r, \mathbf{n})=$ $\sum \varphi^{(k)}(u, \mathbf{n}) r^{-k}$, which gives

$$
\begin{aligned}
& (D-2 k-2) \partial_{u} \varphi^{(k)} \\
& \quad=[\Delta+(k-1)(k-D+2)] \varphi^{(k-1)}+\delta_{k, D-3} \delta(u) \delta(\mathbf{n}, \hat{\mathbf{x}}) .
\end{aligned}
$$

The latter equation is solved by setting $\varphi^{(k)}=0$ for $k \leq$ $\frac{D}{2}-2$ and for $k \geq D-3$, while, for $\frac{D}{2}-1 \leq k \leq D-4$,

$$
\varphi^{(k)}(u, \mathbf{n})=\delta^{(D-4-k)}(u) C_{k}(\mathbf{n}),
$$

where the functions $C_{k}(\mathbf{n})$ are determined recursively by

$$
\begin{aligned}
(D-2 k-2) C_{k}(\mathbf{n}) & =[\Delta+(k-1)(k-D+2)] C_{k-1}(\mathbf{n}), \\
C_{D-4}(\mathbf{n}) & =-(\Delta-D+4)^{-1}(\mathbf{n}, \hat{\mathbf{x}}) .
\end{aligned}
$$

Here, $(\Delta-D+4)^{-1}$ is the Green function for the operator $\Delta-D+4$, which is unique for $D>4$. As a consequence, the field gives rise to the null memory effect 


$$
\begin{aligned}
\left.P_{i}\right|_{u>0}-\left.P_{i}\right|_{u<0} & =\int_{-\infty}^{+\infty} \partial_{i} \varphi d u \\
& =-\frac{1}{r^{D-4}} \partial_{i}(\Delta-D+4)^{-1}(\mathbf{n}, \hat{\mathbf{x}})
\end{aligned}
$$

(note that only the term with $k=D-4$ contributes), consisting in a kick along a direction tangent to the celestial sphere.

\section{Comments on the odd-dimensional case}

In odd dimensions $D \geq 3$ the retarded propagator is given by [48]

$$
G_{D}(x)=c\left(-x^{2}\right)_{+}^{1-\frac{D}{2}} \theta\left(x^{0}\right)
$$

where $c^{-1}=2 \pi^{\frac{D}{2}-1} \Gamma\left(2-\frac{D}{2}\right)$, while $(\kappa)_{+}^{\alpha}$ is the distribution defined as

$$
\left\langle(\kappa)_{+}^{\alpha}, \chi(\kappa)\right\rangle=\int_{0}^{\infty} \kappa^{\alpha} \chi(\kappa) d \kappa \quad \text { for } \alpha>-1
$$

$\chi(\kappa)$ denoting a generic test function, and analytically continued to any $\alpha \neq-1,-2,-3, \ldots$, by

$$
\begin{aligned}
\left\langle(\kappa)_{+}^{\alpha}, \chi(\kappa)\right\rangle= & \frac{(-1)^{n}}{(\alpha+1)(\alpha+2) \cdots(\alpha+n)} \\
& \times\left\langle(\kappa)_{+}^{\alpha+n}, \chi^{(n)}(\kappa)\right\rangle \text { for } n>-1-\alpha .
\end{aligned}
$$

A relevant feature of the wave propagator in odd dimensional spacetimes is that its support is not localized on the light cone $|t|=r$, in contrast with the case of even dimensions, as it is nonzero also for $|t|>r$. This is to be interpreted as the fact that even an ideally sharp perturbation, $\delta(t, \mathbf{x})$, will not give rise to an ideally sharp wave front, but rather the induced radiation will display a dispersion phenomenon and nontrivial disturbances will linger on even after the first wave front has passed.

The solution to Eq. (B1) is then furnished by

$$
\varphi(t, \mathbf{x})=\frac{c q}{2}\left\langle\kappa_{+}^{1-D / 2}, \theta\left(t-\sqrt{\kappa+|\mathbf{x}|^{2}}\right) / \sqrt{\kappa+|\mathbf{x}|^{2}}\right\rangle .
$$

Integrating by parts, and assuming $t>r=|\mathbf{x}|$ (otherwise the field vanishes by causality), one obtains the following expansion:

$$
\begin{aligned}
\frac{2}{c q} \varphi= & \frac{\left(t^{2}-r^{2}\right)^{2-\frac{D}{2}}}{\left(2-\frac{D}{2}\right) t}+\frac{\left(t^{2}-r^{2}\right)^{3-\frac{D}{2}}}{\left(2-\frac{D}{2}\right)\left(3-\frac{D}{2}\right) t^{3}} \frac{1}{2} \\
& +\frac{\left(t^{2}-r^{2}\right)^{4-\frac{D}{2}}}{\left(2-\frac{D}{2}\right)\left(3-\frac{D}{2}\right)\left(4-\frac{D}{2}\right) t^{5}} \frac{1}{2} \cdot \frac{3}{2}+\cdots \\
& +\frac{\left(t^{2}-r^{2}\right)^{-\frac{1}{2}}}{\left(2-\frac{D}{2}\right)\left(3-\frac{D}{2}\right) \cdots\left(-\frac{3}{2}\right)\left(-\frac{1}{2}\right) t^{D-4}} \frac{1}{2} \cdot \frac{3}{2} \cdots\left(\frac{D}{2}-3\right) \\
& +(-1)^{\frac{D-3}{2}} \int_{0}^{t^{2}-r^{2}} \frac{d \kappa}{\sqrt{\kappa}\left(\kappa+r^{2}\right)^{\frac{D}{2}-1}} .
\end{aligned}
$$

Moving to retarded coordinates, this result can be recast as

$$
\varphi(u, r)=\bar{\varphi}(u, r)+\frac{c q}{2}(-1)^{\frac{D-3}{2}} \theta(u) \int_{0}^{u(u+2 r)} \frac{d \kappa}{\sqrt{\kappa}\left(\kappa+r^{2}\right)^{\frac{D}{2}-1}},
$$

where $\bar{\varphi}(u, r)$ is given by a sum of terms proportional to

$$
\frac{\theta(u)}{(u(u+2 r))^{\alpha}(u+r)^{\beta}},
$$

with $\alpha, \beta$ positive and $\alpha+\beta$ half odd. In particular, it is then clear that the limit of this field as $r \rightarrow \infty$ for any fixed $u$ does not display any term with the Coulombic behavior $r^{3-D}$ and hence that there is no memory effect on $\mathcal{I}^{+}$to that order, since

$$
\int_{0}^{u(u+2 r)} \frac{d \kappa}{\sqrt{\kappa}\left(\kappa+r^{2}\right)^{\frac{D}{2}-1}} \sim \frac{1}{r^{D-3}} \int_{0}^{\frac{2 u}{r}} \frac{d x}{\sqrt{x}(1+x)^{\frac{D}{2}-1}} \sim \frac{2 \sqrt{2 u}}{r^{D-\frac{5}{2}}} .
$$

Considering instead the limit of $\varphi$ as $t \rightarrow+\infty$ for fixed $r$, one sees that only the last term in (B31) survives and yields

$$
\begin{aligned}
& \frac{c q}{2}(-1)^{\frac{D-3}{2}} \int_{0}^{\infty} \frac{d \kappa}{\sqrt{\kappa}\left(\kappa+r^{2}\right)^{\frac{D}{2}-1}} \\
& \quad=\frac{c q(-1)^{\frac{D-3}{2}}}{2} \frac{1}{r^{D-3}} B\left(\frac{D-3}{2}\right)=\frac{q}{(D-3) \Omega_{D-2} r^{D-3}} .
\end{aligned}
$$

This means that the Coulombic energy due to the newly created particle is felt by the test charge only at $i^{+}$, namely after one has waited (for an infinite time) at a fixed distance $r$ that the perturbations due to the dispersion occurring in odd spacetime dimensions have died out. To some extent, this is to be regarded as a smeared-out memory effect, as opposed to memory effects occurring sharply at $\mathcal{I}^{+}$near $u=0$ in even dimensions (see also [15]).

The situation does not improve if one considers a particle that is created with a nonzero velocity $\mathbf{v}$. Indeed, boosting the exact solution (B31) by means of (B12), one sees that $\varphi$ goes to zero for fixed $r$ as $t \rightarrow+\infty$. The reason is that, while one waits for the dispersion to die out, the source, 
moving at a constant velocity, has traveled infinitely far from the test charge.

Shifting our attention to the case of null memory, we see that it is possible to provide the following formal solution to the recursion relations (B23), which hold in any dimension. We consider $\varphi=\sum \varphi^{(k)} r^{-k}$, setting $\varphi^{(k)}=0$ for $k \geq D-3$, while, for $k \leq D-4$,

$$
\varphi^{(k)}(u, \mathbf{n})=\delta^{(D-4-k)}(u) C_{k}(\mathbf{n}),
$$

with the functions $C_{k}(\mathbf{n})$ determined recursively by

$$
\begin{aligned}
(D-2 k-2) C_{k}(\mathbf{n}) & =[\Delta+(k-1)(k-D+2)] C_{k-1}(\mathbf{n}), \\
C_{D-4}(\mathbf{n}) & =-(\Delta-D+4)^{-1}(\mathbf{n}, \hat{\mathbf{x}}) .
\end{aligned}
$$

Thus, although the field is highly singular at $u=0$, the resulting null memory effect will be formally identical to the one occurring in even dimensions.

\section{APPENDIX C: EXACT SOLUTION OF $\square \epsilon=0$}

The closed-form solution of the wave equation (3.2) with the boundary condition

$$
\lim _{r \rightarrow \infty} \epsilon(u, r, \mathbf{n})=\epsilon^{(0)}(u, \mathbf{n})
$$

introduced in (5.1) in any even dimension $D$ is given by

$\epsilon(x)=\frac{\Gamma(D-2)}{\pi^{\frac{D-2}{2}} \Gamma\left(\frac{D-2}{2}\right)} \operatorname{Re} \oint \frac{\left(-x^{2}\right)^{\frac{D-2}{2}}}{(-2 x \cdot q+i \varepsilon)^{D-2}} \epsilon^{(0)}(\mathbf{q}) d \Omega(\mathbf{q})$,

where $q=(1, \mathbf{q})$ and the limit $\varepsilon \rightarrow 0^{+}$is understood. The introduction of this small imaginary part is needed in order to avoid the singularities occurring in the angular integration for $|t|<|\mathbf{x}|$, namely outside the light cone. Indeed, it is straightforward to verify that, for any value of $D$ even or odd,

$$
\square \frac{\left(-x^{2}\right)^{\frac{D-2}{2}}}{(-2 x \cdot q)^{D-2}}=0,
$$

while, aligning $\mathbf{n}$ along the $(D-1)$ th direction, we have

$$
\begin{aligned}
\operatorname{Re} & \oint \frac{\left(-x^{2}\right)^{\frac{D-2}{2}}}{(-2 x \cdot q+i \varepsilon)^{D-2}} \epsilon^{(0)}(\mathbf{q}) d \Omega(\mathbf{q}) \\
= & \operatorname{Re} \int_{0}^{\pi} d \theta(\sin \theta)^{D-3} \oint d \Omega^{\prime}\left(\mathbf{q}^{\prime}\right) \\
& \times \frac{2^{2-D}[u(u+2 r)]^{\frac{D-2}{2}}}{[u+r(1-\cos \theta)+i \varepsilon]^{D-2}} \epsilon^{(0)}\left(\mathbf{q}^{\prime} \sin \theta, \cos \theta\right),
\end{aligned}
$$

where $d \Omega^{\prime}\left(\mathbf{q}^{\prime}\right)$ denotes the integral measure on the $(D-3)$ sphere. Letting $\tau=r(1-\cos \theta) / u$, for $u \neq 0$, the previous expression becomes

$$
\begin{aligned}
& \operatorname{Re} \int_{0}^{2 r / u} \frac{u d \tau}{r} \frac{\left(1+\frac{2 r}{u}\right)^{\frac{D-2}{2}}\left(\frac{2 u \tau}{r}-\frac{u^{2} \tau^{2}}{r^{2}}\right)^{\frac{D-4}{2}}}{2^{D-2}(1+\tau+i \varepsilon)^{D-2}} \\
& \quad \times \oint d \Omega^{\prime}\left(\mathbf{q}^{\prime}\right) \epsilon^{(0)}\left(\mathbf{q}^{\prime} \sqrt{\frac{2 u \tau}{r}-\frac{u^{2} \tau^{2}}{r^{2}}}, 1-\frac{u \tau}{r}\right),
\end{aligned}
$$

which, as $r \rightarrow \infty$, tends to

$$
\begin{aligned}
& \frac{1}{2} \operatorname{Re} \int_{0}^{u \cdot \infty} \frac{\tau^{\frac{D-4}{2}} d \tau}{(1+\tau+i \varepsilon)^{D-2}} \oint d \Omega^{\prime}\left(\mathbf{q}^{\prime}\right) \epsilon^{(0)}(\mathbf{n}) \\
& \quad=\frac{\pi^{\frac{D-2}{2}} \Gamma\left(\frac{D-2}{2}\right)}{\Gamma(D-2)} \epsilon^{(0)}(\mathbf{n})
\end{aligned}
$$

for even $D$. The solution (C2) which, to the best of our knowledge, was not previously exhibited in closed form in the literature, is thus compatible with the asymptotic expansion (5.4) and generalizes the expression given in $[30,38]$.

In addition, being nonperturbative, it allows one to explicitly verify the antipodal matching condition

$$
\lim _{r \rightarrow \infty} \epsilon(u=v-2 r, r, \mathbf{n})=\epsilon^{(0)}(-\mathbf{n})
$$

for any fixed advanced time $v$.

\section{APPENDIX D: SOFT PHOTON THEOREM IN EVEN $D$}

We would like to show that the surface charges defined in Sec. V B enter Weinberg's soft theorem [49,50]. More precisely, we will see how the Weinberg theorem implies the validity of the Ward identities associated with such charges in $D \geq 4[34,35]$.

The surface charge associated with (5.4), evaluated at $\mathcal{I}_{-}^{+}$, reads

$\mathcal{Q}_{\epsilon}=\int_{\mathcal{I}_{ \pm}^{+}}\left(\partial_{u} A_{r}^{(D-2)}+(D-3) A_{u}^{(D-3)}\right) \epsilon^{(0)} d \Omega_{D-2}$,

where we have taken into account the absence of radiation terms for $u \rightarrow-\infty$. Recasting (D1) as an integral over the whole of $\mathcal{I}^{+}$, and assuming that no contribution arises at $\mathcal{I}_{+}^{+}$, which is the case in particular if there are no stable massive charges, we find

$$
\mathcal{Q}_{\epsilon}=-\frac{1}{r^{D-4}} \int_{\mathcal{I}^{+}} \partial_{u}^{2} A_{r}^{(D-2)} \epsilon^{(0)} d u d \Omega_{D-2},
$$

where we have used the fact that, after recursive gauge fixing, $A_{u}^{(D-3)}$ is independent of $u$ on shell. We would like to express (D2) in terms of the leading radiation field, which, as we shall see below, indeed contains the creation and annihilation operator of asymptotic photons. To this end, we first combine (3.8) and (3.5) and obtain 


$$
\begin{aligned}
\mathcal{D} \cdot A^{(k-1)}= & \frac{\Delta-(D-2-k)(D-3-k)}{D-2-2 k} A_{r}^{(k)} \\
& +(D-3-k) A_{u}^{(k)} ;
\end{aligned}
$$

employing (3.7) as well,

$$
\partial_{u} A_{r}^{(k+1)}=\mathscr{D}_{k} A_{r}^{(k)}-A_{u}^{(k)},
$$

where $\mathscr{D}_{k}$ is given in (4.21).

Employing this relation recursively, we find

$$
\partial_{u}^{D / 2} A_{r}^{(D-2)}=\prod_{l=D / 2}^{D-3} \mathscr{D}_{l} \partial_{u} \mathcal{D} \cdot A^{\left(\frac{D-4}{2}\right)},
$$

where we have used (3.8) to deduce $\partial_{u} A_{r}^{\left(\frac{D}{2}\right)}=\mathcal{D} \cdot A^{\left(\frac{D-4}{2}\right)}$. In the above writing, we adopt the convention that for $D=4$ the product $\prod_{l} \mathscr{D}_{l}$ (which in this case has a formally illdefined range) reduces to the identity. We can then use (D5) to recast (D2) as

$$
\begin{aligned}
\mathcal{Q}_{\epsilon}= & -\frac{1}{r^{D-4}} \int_{-\infty}^{+\infty}\left(\int_{-\infty}^{u} d u\right)^{D / 2-2} \partial_{u} \mathcal{D} \cdot A^{\left(\frac{D-4}{2}\right)} \\
& \times \prod_{l=D / 2}^{D-3} \mathscr{D}_{l} \epsilon^{(0)} d u d \Omega_{D-2} .
\end{aligned}
$$

On the other hand, the asymptotic expansion of the free electromagnetic field operator, expressed in terms of creation and annihilation operators, yields, to leading order,

$$
\begin{aligned}
\mathcal{A}_{i}\left(u, r, x^{k}\right)= & \frac{i^{1-D / 2}}{8 \pi^{2} r^{(D-4) / 2}} \int_{0}^{+\infty}\left(\frac{\omega}{2 \pi}\right)^{(D-4) / 2} \\
& \times e^{-i \omega u} \epsilon_{i}^{\sigma}(\hat{x}) a_{\sigma}(\omega \hat{x}) d \omega+\text { H.c. },
\end{aligned}
$$

where $\hat{x}^{\mu}=x^{\mu} / r$, while $\epsilon^{\sigma}$ are polarization tensors for the $D-2$ propagating helicities. This formula thus provides an explicit expression for $A_{i}^{\left(\frac{D-4}{2}\right)}$ and hence allows us to make explicit the relation between the charge $\mathcal{Q}_{\epsilon}$ and the soft photon creation and annihilation operators as follows (we employ the prescription $\left.\int_{-\infty}^{+\infty} d u \int_{0}^{+\infty} d \omega e^{i \omega u} f(\omega)=\lim _{\omega \rightarrow 0^{+}} f(\omega) / 2\right)$ :

$$
\begin{aligned}
\mathcal{Q}_{\epsilon}= & \frac{1}{8(2 \pi)^{(D-2) / 2} r^{D-4}} \lim _{\omega \rightarrow 0^{+}} \int_{S^{D-2}} \mathcal{D}^{i}\left[\epsilon_{i}^{\sigma}(\hat{x}) \omega a_{\sigma}(\omega \hat{x})+\text { H.c. }\right] \\
& \times \prod_{l=D / 2}^{D-3} \mathscr{D}_{l} \epsilon^{(0)}(\hat{x}) d \Omega_{D-2}(\hat{x}) .
\end{aligned}
$$

Assuming that the charge $\mathcal{Q}_{\epsilon}$, together with its counterpart at $\mathcal{I}^{-}$, generates the residual symmetry $\delta \psi(u, r, \hat{x})=$ $i \epsilon^{(0)}(\hat{x})+\mathcal{O}\left(r^{-1}\right)$ in a canonical way, and employing suitable antipodal matching and crossing symmetry conditions, we have the Ward identity

$$
\begin{aligned}
& \frac{1}{2(2 \pi)^{(D-2) / 2}} \int_{S^{D-2}} \epsilon_{i}^{\sigma}(\hat{x}) \lim _{\omega \rightarrow 0^{+}} \mathcal{D}^{i}\left\langle\text { out }\left|\omega a_{\sigma}(\omega \hat{x}) \mathcal{S}\right| \text { in }\right\rangle \\
& \times \prod_{l=D / 2}^{D-3} \mathscr{D}_{l} \epsilon^{(0)}(\hat{x}) d \Omega_{D-2}(\hat{x}) \\
& =\sum_{n} e_{n} \epsilon^{(0)}\left(\hat{x}_{n}\right)\langle\text { out }|\mathcal{S}| \text { in }\rangle,
\end{aligned}
$$

where the sum on the right-hand side extends to all charged external particles in the amplitude and $e_{n}$ is the electric charge of the $n$th particle (taking into account with a suitable sign whether the particle is outgoing or incoming, respectively). Notably, the left-hand side contains exactly the combination $\mathcal{P}[\cdot]=\lim _{\omega \rightarrow 0^{+}}[\omega \cdot]$ that selects the pole in the amplitude with the soft insertion.

On the other hand, the Weinberg theorem for an amplitude involving external massless particles with momenta $p_{n}=E_{n}\left(1, \hat{x}_{n}\right)$ and a soft photon emitted with helicity $\sigma$ pointing along the $\hat{n}$ direction on the celestial sphere reads

$$
\lim _{\omega \rightarrow 0^{+}}\left\langle\text {out }\left|\omega a_{\sigma}(\omega \hat{n}) \mathcal{S}\right| \text { in }\right\rangle=\sum_{n} \frac{e_{n} \epsilon^{\sigma}(\hat{n})^{*} \cdot p_{n}}{p_{n} \cdot(1, \hat{n})}\langle\text { out }|\mathcal{S}| \text { in }\rangle .
$$

Multiplying this relation by $\epsilon_{i}^{\sigma}(\hat{n})$ and summing over $\sigma$, we see that this is equivalent to

$\epsilon_{i}^{\sigma}(\hat{n}) \lim _{\omega \rightarrow 0^{+}}\left\langle\right.$out $\left|\omega a_{\sigma}(\omega \hat{n}) S\right|$ in $\rangle=\sum_{n} e_{n} \mathcal{D}_{i} \alpha\left(\hat{x}_{n}, \hat{n}\right)\langle$ out $|S|$ in $\rangle$,

where we have used the completeness relation for polarization vectors and defined a function

$$
\alpha(\hat{x}, \hat{n})=\log (1-\hat{x} \cdot \hat{n}) .
$$

This function $\alpha$ satisfies the following identity (see [24]):

$$
\frac{1}{2(2 \pi)^{(D-2) / 2}} \prod_{l=D / 2}^{D-3} \mathscr{D}_{l} \Delta \alpha(\hat{x}, \hat{n})=\delta(\hat{x}, \hat{n}),
$$

where $\hat{x}$ is here treated as a constant vector on the unit sphere and $\delta(\hat{x}, \hat{n})$ is the invariant delta function on the $(D-2)$-sphere. Now, acting with the differential operator

$$
\frac{1}{2(2 \pi)^{(D-2) / 2}} \prod_{l=D / 2}^{D-3} \mathscr{D}_{l} \cdot \mathcal{D}^{i}
$$

on Eq. (D11), multiplying by an arbitrary $\epsilon^{(D-4)}(\hat{x})$ and integrating over the unit sphere allows one to retrieve the Ward identity (D9), thanks to the relation (D13). 
This proves that the Weinberg factorization implies the existence of the asymptotic symmetry Ward identities.

Remarkably, the charge (3.22) associated with the symmetry (3.21), which is responsible for the memory effect, formally differs from (D1) only by a factor of $1 / r^{D-4}$ (other than by the substitution $\epsilon^{(0)} \leftrightarrow \epsilon^{(D-4)}$ ), which makes it vanish on $\mathcal{I}^{+}$. However, the corresponding symmetry transformation of the matter fields would be $\delta \psi(u, r, \hat{x})=i \epsilon^{(D-4)}(\hat{x}) / r^{D-4}+\mathcal{O}\left(r^{3-D}\right)$, and hence would give rise to Ward identities completely equivalent to (D9), with the factors of $1 / r^{D-4}$ canceling each other on the two sides. This indicates that the large gauge symmetry (5.6) and the residual symmetry (3.21), acting at Coulombic order, can both be seen as consequences of Weinberg's soft theorem. This is also reflected in the observation that the Fourier transform of the soft factor occurring in Weinberg's theorem is strictly related to the memory formulas [10].
[1] Y. B. Zeldovich and A. G. Polnarev, Radiation of gravitational waves by a cluster of superdense stars, Sov. Astron. 18, 17 (1974).

[2] D. Christodoulou, Nonlinear Nature of Gravitation and Gravitational Wave Experiments, Phys. Rev. Lett. 67, 1486 (1991).

[3] L. Bieri and D. Garfinkle, An electromagnetic analogue of gravitational wave memory, Classical Quantum Gravity 30, 195009 (2013).

[4] A. Tolish and R. M. Wald, Retarded fields of null particles and the memory effect, Phys. Rev. D 89, 064008 (2014).

[5] S. Pasterski, Asymptotic symmetries and electromagnetic memory, J. High Energy Phys. 09 (2017) 154.

[6] P. Mao, H. Ouyang, J. B. Wu, and X. Wu, New electromagnetic memories and soft photon theorems, Phys. Rev. D 95, 125011 (2017).

[7] M. Sarkkinen, Memory effect in electromagnetic radiation, Master Thesis, University of Helsinki, 2018, https://helda .helsinki.fi/handle/10138/231490.

[8] N. Jokela, K. Kajantie, and M. Sarkkinen, Memory effect in Yang-Mills theory, Phys. Rev. D 99, 116003 (2019).

[9] D. Garfinkle, S. Hollands, A. Ishibashi, A. Tolish, and R. M. Wald, The memory effect for particle scattering in even spacetime dimensions, Classical Quantum Gravity 34, 145015 (2017).

[10] P. Mao and H. Ouyang, Note on soft theorems and memories in even dimensions, Phys. Lett. B 774, 715 (2017).

[11] Y. Hamada, M. S. Seo, and G. Shiu, Electromagnetic duality and the electric memory effect, J. High Energy Phys. 02 (2018) 046.

[12] G. Satishchandran and R. M. Wald, Memory effect for particle scattering in odd spacetime dimensions, Phys. Rev. D 97, 024036 (2018).

[13] Y. Hamada and S. Sugishita, Notes on the gravitational, electromagnetic and axion memory effects, J. High Energy Phys. 07 (2018) 017.

[14] H. Afshar, E. Esmaeili, and M. M. Sheikh-Jabbari, String memory effect, J. High Energy Phys. 02 (2019) 053.

[15] G. Satishchandran and R. M. Wald, Asymptotic behavior of massless fields and the memory effect, Phys. Rev. D 99, 084007 (2019).

[16] M. Pate, A. M. Raclariu, and A. Strominger, Gravitational memory in higher dimensions, J. High Energy Phys. 06 (2018) 138.
[17] L. Susskind, Electromagnetic memory, arXiv:1507.02584.

[18] M. Pate, A. M. Raclariu, and A. Strominger, Color Memory: A Yang-Mills Analog of Gravitational Wave Memory, Phys. Rev. Lett. 119, 261602 (2017).

[19] A. Ball, M. Pate, A. M. Raclariu, A. Strominger, and R. Venugopalan, Measuring color memory in a color glass condensate at electron-ion colliders, Ann. Phys. (Amsterdam) 407, 15 (2019).

[20] A. Strominger, Asymptotic symmetries of Yang-Mills theory, J. High Energy Phys. 07 (2014) 151.

[21] G. Barnich and P. H. Lambert, Einstein-Yang-Mills theory: Asymptotic symmetries, Phys. Rev. D 88, 103006 (2013).

[22] T. He, P. Mitra, A. P. Porfyriadis, and A. Strominger, New symmetries of massless QED, J. High Energy Phys. 10 (2014) 112.

[23] V. Lysov, S. Pasterski, and A. Strominger, Low's Subleading Soft Theorem as a Symmetry of QED, Phys. Rev. Lett. 113, 111601 (2014).

[24] D. Kapec, V. Lysov, and A. Strominger, Asymptotic symmetries of massless QED in even dimensions, Adv. Theor. Math. Phys. 21, 1747 (2017).

[25] M. Campiglia and A. Laddha, Asymptotic symmetries of QED and Weinberg's soft photon theorem, J. High Energy Phys. 07 (2015) 115.

[26] A. Strominger, Magnetic Corrections to the Soft Photon Theorem, Phys. Rev. Lett. 116, 031602 (2016).

[27] S. G. Avery and B. U.W. Schwab, Noether's second theorem and Ward identities for gauge symmetries, J. High Energy Phys. 02 (2016) 031.

[28] A. Campoleoni, D. Francia, and C. Heissenberg, Asymptotic charges at null infinity in any dimension, Universe 4, 47 (2018).

[29] M. Henneaux and C. Troessaert, Asymptotic symmetries of electromagnetism at spatial infinity, J. High Energy Phys. 05 (2018) 137.

[30] H. Hirai and S. Sugishita, Conservation laws from asymptotic symmetry and subleading charges in QED, J. High Energy Phys. 07 (2018) 122.

[31] V. Hosseinzadeh, A. Seraj, and M. M. Sheikh-Jabbari, Soft charges and electric-magnetic duality, J. High Energy Phys. 08 (2018) 102.

[32] A. Campoleoni, D. Francia, and C. Heissenberg, Asymptotic symmetries and charges at null infinity: From low to high spins, EPJ Web Conf. 191, 06011 (2018). 
[33] R. Gonzo, T. McLoughlin, D. Medrano, and A. Spiering, Asymptotic charges and coherent states in QCD, arXiv: 1906.11763.

[34] T. He and P. Mitra, Asymptotic symmetries and Weinberg's soft photon theorem in Mink $_{d+2}$, arXiv:1903.02608.

[35] T. He and P. Mitra, Asymptotic symmetries in $(d+2)$ dimensional gauge theories, arXiv:1903.03607.

[36] M. Henneaux and C. Troessaert, Asymptotic structure of electromagnetism in higher spacetime dimensions, Phys. Rev. D 99, 125006 (2019).

[37] T. He and P. Mitra, New magnetic symmetries in $(d+2)$ dimensional QED, arXiv:1907.02808.

[38] A. Strominger, Lectures on the Infrared Structure of Gravity and Gauge Theory (Princeton University Press, Princeton, NJ, 2018).

[39] S. K. Wong, Field and particle equations for the classical Yang-Mills field and particles with isotopic spin, Nuovo Cimento A 65, 689 (1970).

[40] B. Kosyakov, Introduction to the Classical Theory of Particles and Fields (Springer-Verlag, Berlin, Heidelberg, 2007).

[41] D. Kapec, V. Lysov, S. Pasterski, and A. Strominger, Higher-dimensional supertranslations and Weinberg's soft Graviton theorem, Ann. Math. Sci. Appl. 2, 69 (2017).
[42] M. Campiglia, L. Freidel, F. Hopfmueller, and R. M. Soni, Scalar asymptotic charges and dual large gauge transformations, J. High Energy Phys. 04 (2019) 003.

[43] D. Francia and C. Heissenberg, Two-form asymptotic symmetries and scalar soft theorems, Phys. Rev. D 98, 105003 (2018).

[44] A. Riello, Soft charges from the geometry of field space, arXiv:1904.07410.

[45] A. Aggarwal, Supertranslations in higher dimensions revisited, Phys. Rev. D 99, 026015 (2019).

[46] L. Freidel, F. Hopfmüller, and A. Riello, Holographic renormalization in flat space: Symplectic potential and charges of electromagnetism, arXiv:1904.04384.

[47] G. Barnich and F. Brandt, Covariant theory of asymptotic symmetries, conservation laws and central charges, Nucl. Phys. B633, 3 (2002).

[48] F. G. Friedlander, The Wave Equation on a Curved SpaceTime (Cambridge University Press, Cambridge, England, 1975).

[49] S. Weinberg, Photons and gravitons in s matrix theory: Derivation of charge conservation and equality of gravitational and inertial mass, Phys. Rev. B 135, 1049 (1964).

[50] S. Weinberg, Infrared photons and gravitons, Phys. Rev. B 140, 516 (1965). 